\title{
Robotic Herding of a Flock of Birds Using an Unmanned Aerial Vehicle
}

\author{
Aditya A. Paranjape (), Soon-Jo Chung (), Senior Member, IEEE, Kyunam Kim (), \\ and David Hyunchul Shim ${ }^{\circledR}$, Senior Member, IEEE
}

\begin{abstract}
In this paper, we derive an algorithm for enabling a single robotic unmanned aerial vehicle to herd a flock of birds away from a designated volume of space, such as the air space around an airport. The herding algorithm, referred to as the $m$ waypoint algorithm, is designed using a dynamic model of bird flocking based on Reynolds' rules. We derive bounds on its performance using a combination of reduced-order modeling of the flock's motion, heuristics, and rigorous analysis. A unique contribution of the paper is the experimental demonstration of several facets of the herding algorithm on flocks of live birds reacting to a robotic pursuer. The experiments allow us to estimate several parameters of the flocking model, and especially the interaction between the pursuer and the flock. The herding algorithm is also demonstrated using numerical simulations.
\end{abstract}

Index Terms-Aerial robotics, biologically inspired robots, field robots, motion control.

\section{INTRODUCTION}

B IRD AND OTHER wildlife collisions with aircraft cause well over $\$ 1.2$ billion in damages to the aviation industry worldwide [1], [2]. The Federal Aviation Administration (FAA) documented 142000 wild-life strikes at U.S. airports between 1990 and 2013, with birds being involved in $97 \%$ of the reported cases [3]. A large number of passive and active methods are currently used for deterring birds from entering the airspace around airports [1], [4], [5], which depend on the type of birds encountered at the airport. Passive techniques typically work by curtailing the food and water resources around the airfield or laying bird-repellent grass swards. On the other hand, active techniques include the use of live ammunition and flares at airports. Usually, a combination of the aforementioned techniques

Manuscript received October 12, 2017; revised April 4, 2018; accepted April 7, 2018. This paper was recommended for publication by Guest Editor P. Dames and Editor F. Park upon evaluation of the reviewers' comments. This work was supported in part by the National Science Foundation CAREER Award NSF IIS 1253758 \& 1664186 and in part by the California Institute of Technology. (Corresponding author: Soon-Jo Chung.)

A. A. Paranjape is with the Department of Aeronautics, Imperial College London, London SW7 2AZ, U.K. (e-mail: a.paranjape@imperial.ac.uk).

S.-J. Chung and K. Kim are with the Graduate Aerospace Laboratories, California Institute of Technology, Pasadena, CA 91125 USA (e-mail: sjchung@caltech.edu; knkim@caltech.edu).

D. H. Shim is with the Department of Electrical Engineering, Korea Advanced Institute of Science and Technology, Daejeon 305-701, South Korea (e-mail: hcshim@kaist.ac.kr).

This paper has supplementary downloadable material available at http://ieeexplore.ieee.org.

Color versions of one or more of the figures in this paper are available online at http://ieeexplore.ieee.org.

Digital Object Identifier 10.1109/TRO.2018.2853610
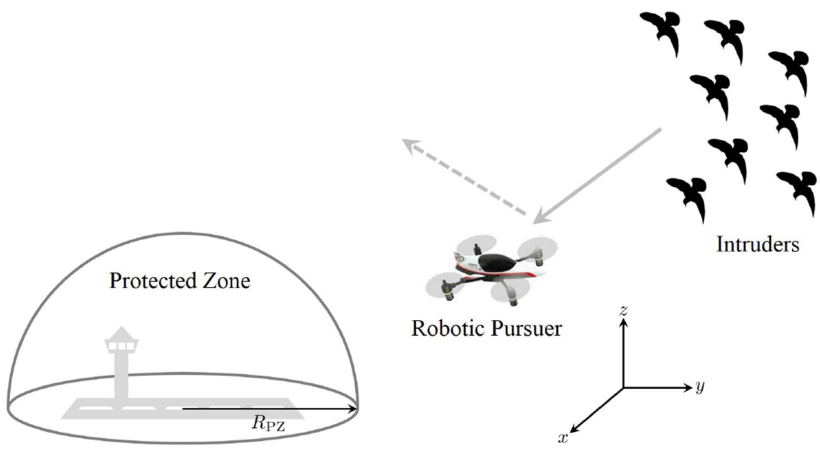

Fig. 1. Setting for the problem addressed in the paper.

is required for effective bird control, and even that effectiveness is known to be limited [4]. The only proven lasting way is to use birds of prey [5], but it is considerably difficult to train and control real birds. Further, the most effective performers (e.g., peregrine falcons) are endangered species. As an alternative, remote-controlled airplanes resembling birds of prey have been tested using skilled human pilots [6].

In this paper, we develop a novel robotic herding algorithm for birds flying near airports using unmanned aerial vehicles (UAVs). The present paper is based on the preliminary work reported in [7], [8], and differs in its analysis of the stability of flocks, the analysis of the performance of herding algorithms, and the experimental demonstration of the principles underlying the herding algorithm. A graphical prelude to this paper is depicted in Fig. 1.

\section{A. Overview of the Literature}

Modeling the behavior of flocks is the starting point for the development of herding algorithms. Reynolds [9] formulated simple motion behaviors (collision avoidance, velocity matching, and flock centering) for creating computer animations of bird flocks and simulating the stable collective motion seen therein. Reynolds' model has formed the basis for other flocking models such as the self-propelled particle model [10], [11] and a leader-based modification of Reynolds' model [12]. Alongside theoretical modeling, attempts have been made to extract flocking rules, aggregate patterns, and ordering from empirical studies on large flocks of birds and fish [13], [14]. The reader is referred to [15] for a detailed review of collective animal behavior. 
Over the years, several distributed, scalable coordination algorithms have been developed while trying to obtain mathematical guarantees on the stability of the formation [16]-[22]. This has been accompanied by parallel work on understanding the presence of order and phase transitions in flocks [23], building upon analogies between flocks on the one hand, and fluid and magnetic systems on the other. Flocking dynamics described by Reynolds' rule are known to be asymptotically stable under fairly weak conditions on the topology of the underlying graph [17], [18], [24], [25]. Stronger results, such as exponential stability, have been found for linear time-varying consensus protocol for single integrator systems [26], in the context of synchronization for second-order Euler-Lagrange systems [20]-[22], and using tools from dynamical systems theory for time-invariant, undirected graph topologies in second-order, two-dimensional (2-D) flocking dynamics [27]. Preliminary results on exponential stability of flocks under tree and star topology constraints are presented in [8].

Modeling the response of a flock to external pursuers is essential for developing herding models and strategies. Evasive strategies used by flocks against one or more predators are presented in [28], [29]. Escape waves, as a means for formulating the evasive response, have been examined for starling flocks [30] and fish schools [31]. A simulation study of agitation waves in starling flocks is presented in [32]. The dynamics between a school of fish and a pursuer are shown to depend on local, fasttime scale predator-prey interactions [33]. The expected size of flocks is correlated with the likelihood of predation: Compact and large flocks occur in areas with higher predation, and vice-versa [34].

Early work on herding algorithms used geometric principles to identify the relative position and posture of the shepherd with respect to the flock [35]-[37], in order to first confine the flock within a well-defined spatial zone and then impose trajectory tracking on the confined flock [38], [39]. It is generally well known that these algorithms may perform poorly when a single shepherd is employed [37]. Heuristic herding strategies used by solitary sheep dogs were modeled in [40] and used to design a strategy that enforces confinement to achieve herding. However, the design was not analyzed rigorously, which leaves open the question of its limitations and performance guarantees.

\section{B. Objectives and Contributions}

The primary objective of this paper is to design a strategy for diverting and herding a flock of birds away from a specified region. This region is modeled conservatively as a cylindrical volume around an airport located on the ground. Our algorithm can be extended readily to the case where the specified region is spherical, as depicted in Fig. 1, rather than cylindrical.

We present a novel herding technique, called the $m$-waypoint herding algorithm. The robotic UAV interacts with the flock by positioning itself sequentially at a periodically refreshed set of $m$ points. It relies on the inherent stability of the flocking dynamics to prevent fragmentation of the flock, and the $m$ points are chosen to maximise the deflection of the flock's flight path. We demonstrate that the algorithm also leads to a reduction in the physical space occupied by the flock, measured in terms of its radius. Importantly, the technique works effectively with a single robotic UAV, and can be generalized readily for multiple UAVs.

An important contribution of the paper is the series of experiments performed on live birds in order to identify some of the parameters of the flocking dynamic model and identify the nature of the evasive action taken by birds in response to a robotic pursuer. These are important steps in validating the design premise of the $m$-waypoint herding algorithm. The herding algorithm is demonstrated through simulations on a flocking model similar to that estimated from the experiments.

The problem addressed in the paper is similar to [36]. A major difference, though, is a combination of the rigorous analysis accompanying the algorithm and the experimental demonstration on live birds. Another difference is the non-zero minimum speed of the flock, which is a consequence of the birds needing a certain minimum speed to stay aloft.

We use a standard flocking model, similar to Reynolds [9], [17], augmented with evasion laws similar to [28], [29]. It is assumed that the pursuer has access to real-time data about the flock, such as that provided by avian radars [41].

\section{Organization}

The paper is organized as follows. In Section II, we present the problem formulation. Conditions for exponential stability of flocks are derived in Section III. The pursuer-flock interaction is studied analytically in Section IV, and the $m$-waypoint herding algorithm is presented in Section V. The performance of the algorithm is analyzed rigorously in Section VI. Experiments conducted on live flocks are reported in Section VII, while numerical simulations are described in Section VIII.

\section{PROBLEM FoRMULATION}

\section{A. Preliminaries}

Consider a flock of $n$ (usually $\gg 1$ ) birds. We denote the position and the velocity of the $i$ th bird by $\boldsymbol{x}_{i} \in \mathbb{R}^{3}$ and $\boldsymbol{v}_{i} \in \mathbb{R}^{3}$, respectively. The vector between the $i$ th and $j$ th birds is denoted by $\mathbf{r}_{i j}=\boldsymbol{x}_{j}-\boldsymbol{x}_{i}$. We use the subscript "p" for the pursuer, so that $\boldsymbol{x}_{p}$ denotes its position vector, and $\mathbf{r}_{p i}=\boldsymbol{x}_{i}-\boldsymbol{x}_{p}$ is the vector from the pursuer to the $i$ th bird. Given a vector $\mathbf{r}$, we denote unit vector along $\mathbf{r}$ by $\hat{\mathbf{r}}$.

Let $R_{\text {com }}$ denote the communication range for the interaction between two birds. The neighborhood of the $i$ th bird is defined using the standard Euclidean distance as

$$
\mathcal{N}_{i}=\left\{j \leq n:\left\|\boldsymbol{x}_{i}-\boldsymbol{x}_{j}\right\| \leq R_{\mathrm{com}}\right\}
$$

and it is assumed that two birds interact only if their distance is less than or equal to $R_{\text {com. }}$ The set of birds $(\mathcal{V})$, thus, forms a graph $\mathcal{G}=(\mathcal{V}, \mathcal{E})$ where

$$
\begin{aligned}
& \mathcal{V}=\{1,2, \ldots, n\} \\
& \mathcal{E}=\left\{(i, j) \in \mathcal{V} \times \mathcal{V} \mid j \in \mathcal{J}_{i} \subseteq \mathcal{N}_{i}\right\}, \operatorname{card}(\mathcal{E})=p .
\end{aligned}
$$

Note that if $\mathcal{J}_{i}=\mathcal{N}_{i}$ for all $i$, the graph is undirected. Otherwise, it is directed. The incidence matrix of the graph is denoted as 


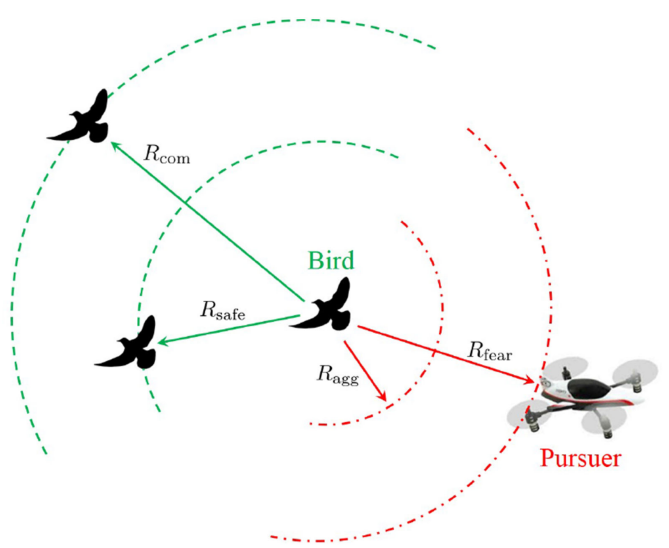

Fig. 2. Important distance variables in the flocking model. Green dashed lines are distances related to neighboring birds and red dash-dot lines are distances related to the pursuer. Note that $R_{\mathrm{com}}$ and $R_{\text {fear }}$ are unrelated. $R_{\mathrm{agg}}$ is smaller than $R_{\text {fear }}$.

$B_{c} \in \mathbb{R}^{n \times p}$. Recall that the $(i, j)$ th entry of $B_{c}$ is 1 if the $j$ th edge is incoming at $i,-1$ if outgoing at $i$, and 0 otherwise. An undirected edge is treated as two separate directed edges in this formulation of $B_{c}$. We denote by $\Delta(\boldsymbol{\nu})$ the diagonal matrix formed by the elements of the tuple $\nu$. Finally, we define the centroid and the radius of the flock as follows:

$$
\boldsymbol{x}_{c g}=\frac{1}{n} \sum_{i=1}^{n} \boldsymbol{x}_{i}, R_{F} \triangleq \max _{i}\left\|\boldsymbol{x}_{i}-\boldsymbol{x}_{c g}\right\| .
$$

\section{B. Flock Dynamics}

The dynamics of an individual ( $i$ th) bird are described by a nonlinear, second-order differential equation based on Reynolds' rules [9] and augmented with an evasive response

$$
\begin{aligned}
\dot{\boldsymbol{x}}_{i}= & \boldsymbol{v}_{i} \\
\dot{\boldsymbol{v}}_{i}= & k_{s} \sum_{j \in \mathcal{J}_{i}}\left(1-\frac{R_{\text {safe }}^{3}}{\left\|\mathbf{r}_{i j}\right\|^{3}}\right) \mathbf{r}_{i j}+k_{a} \sum_{j \in \mathcal{J}_{i}}\left(\boldsymbol{v}_{j}-\boldsymbol{v}_{i}\right) \\
& +k_{g}\left(\boldsymbol{v}_{d}-\boldsymbol{v}_{i}\right)+k_{p} \boldsymbol{H}\left(\mathbf{r}_{p i}\right)
\end{aligned}
$$

where $R_{\text {safe }}$ denotes the steady-state distance between two adjacent members of the flock; $\boldsymbol{v}_{d}$ denotes the preselected, steady velocity of each member of the flock; and $\boldsymbol{H}\left(\mathbf{r}_{p i}\right)=\boldsymbol{H}\left(\boldsymbol{x}_{i}-\boldsymbol{x}_{p}\right)$ denotes the evasive response to the pursuer. We assume that $\boldsymbol{v}_{d}$ lies in the horizontal plane.

Fig. 2 summarizes the important distance variables that appear in the model. The terms $R_{\text {safe }}$ and $R_{\text {com }}$ have been defined already. The terms $R_{\text {fear }}$ and $R_{\text {agg }}\left(<R_{\text {fear }}\right)$ refer to critical distances in the bird-pursuer interaction, and we assume that both of these quantities are known for a given flock. The flock responds to a pursuer only when the pursuer is within a radius $R_{\text {fear }}$ of a member of the flock. Within $R_{\text {fear }}$, the evasive behavior takes two different forms.

1) If the pursuer is within $\left[R_{\mathrm{agg}}, R_{\mathrm{fear}}\right]$, the bird tries to accelerate radially away from the pursuer.

2) If the pursuer is within $R_{\mathrm{agg}}$, the bird tries to out-maneuver the pursuer by turning and/or climbing rapidly.

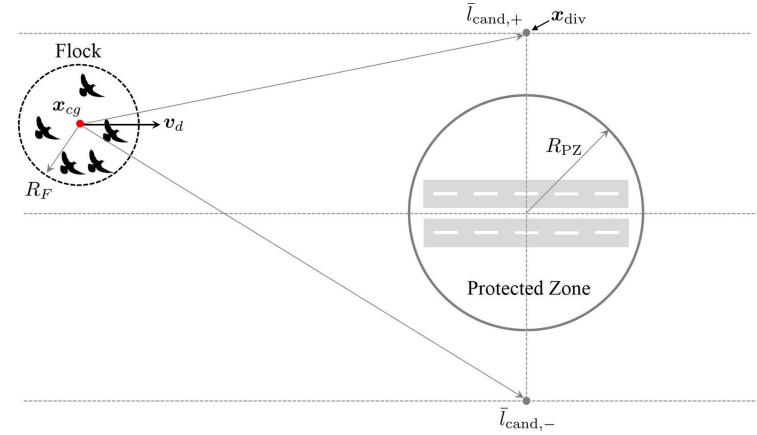

Fig. 3. Example showing the two loci of candidate target points, and the chosen target point $\boldsymbol{x}_{\text {div }}$. Being vertical lines, the loci appear as points in this top view.

The behavior detailed above was observed in experiments with live birds, which are described in Section VII. Since the out-turning or 3-D behavior can have adverse implications for the stability of the flock, we treat $R_{\mathrm{agg}}$ as a lower bound for the permissible distance between the pursuer and the flock. We model $\boldsymbol{H}(\cdot)$ as follows:

$$
\boldsymbol{H}\left(\mathbf{r}_{p i}\right)= \begin{cases}\frac{\mathbf{r}_{p i}}{\left\|\mathbf{r}_{p i}\right\|^{3}}, & \text { if }\left\|\mathbf{r}_{p i}\right\|<R_{\text {fear }} \text { and } \boldsymbol{r}_{p i}^{\top} \dot{\boldsymbol{r}}_{p i}<0 \\ 0 & \text { otherwise }\end{cases}
$$

and we impose the restriction that $\left\|\boldsymbol{r}_{p i}\right\| \geq R_{\mathrm{agg}}+\epsilon$ for all $i$, where $\epsilon>0$ is an arbitrarily chosen constant.

\section{Herding Objectives}

The spatial volume that needs to be kept free of birds is referred to, hereafter, as the protected zone (PZ). We assume that $\mathrm{PZ}$ is cylindrical with radius $R_{\mathrm{PZ}}$. We assume that the birds fly along a constant, preselected heading, $\hat{\boldsymbol{v}}_{d}$, when not disturbed by a pursuer. This is true, for instance, for flocks flying to migration grounds considerably far from PZ. It is clear that the pursuer only needs to move the flock to a point from which its flight path (along $\hat{\boldsymbol{v}}_{d}$ ) would no longer cross PZ. We denote this target point as $\boldsymbol{x}_{\mathrm{div}}(t)$, noting that its exact position may change with time as described presently.

We define the global coordinate frame with its origin placed at the centre of PZ, and its axes pointing north, east, and up, respectively. Let $h(t)$ denote the instantaneous altitude of the flock. We start by constructing two vertical lines (in the global frame), which serve as loci for all possible target points. Recall that $\boldsymbol{v}_{d}$ in (4) is the predetermined nominal flock velocity, which satisfies $\hat{\mathbf{k}}^{\top} \boldsymbol{v}_{d}=0$ where $\hat{\mathbf{k}}$ is the unit vector pointing upwards in the global frame. We define the unit vector $\hat{\boldsymbol{v}}_{d}^{\perp}$, which satisfies $\boldsymbol{v}_{d}^{\top} \hat{\boldsymbol{v}}_{d}^{\perp}=\hat{\mathbf{k}}^{\top} \hat{\boldsymbol{v}}_{d}^{\perp}=0$. The two candidate loci are then given by $\bar{l}_{\text {cand }, \pm}(a)= \pm\left(R_{\mathrm{PZ}}+s\right) \hat{\boldsymbol{v}}_{d}^{\perp}+a \hat{\mathbf{k}}$ where $a \in \mathbb{R}$, and $s>R_{F}$ is a suitably chosen constant. We denote $R_{\text {div }} \triangleq R_{\mathrm{PZ}}+s$. Of these candidate loci, we select the one closest to $\boldsymbol{x}_{c g}$ at $t=0$ (the moment at which the herding commences), as illustrated in Fig. 3. Finally, at each $t$, we set the instantaneous target waypoint on the chosen locus as $\boldsymbol{x}_{\mathrm{div}}=(+/-) R_{\mathrm{div}} \hat{\boldsymbol{v}}_{d}^{\perp}+h(t) \hat{\mathbf{k}}$. If the two loci are equidistant from $\boldsymbol{x}_{c g}$, then any of the two loci can be chosen. However, this choice persists for the remainder of the herding operation. This selection of herding target points is a 
logical extension from 2-D to 3-D when the herding algorithm does not seek to control the altitude of the flock.

The objective of the herding algorithm is to prescribe a trajectory $\boldsymbol{x}_{p}(t)$ for the pursuer, which enables it to do the following functions.

1) Move the center of gravity (CG) of the flock to $x_{\text {div }}$ in finite time.

2) Avoid fragmentation of the flock.

3) Keep the flock outside the PZ during the course of herding.

\section{Stability ANALYSIS OF THE Flock}

In this section, we determine the conditions for exponential stability of the flocking dynamics. These conditions can be used to select the gains and the underlying graph in (4) for analytical investigation when sufficient empirical data is not available to determine these parameters. The resulting robustness of the flock implies that the pursuer is free to approach the flock from any direction. As long as it maintains a certain minimum distance between itself and the flock (see Section IV), the flock will not undergo fragmentation.

\section{A. Snapshot Dynamics, Steady States, and Linearization}

We define two column vectors $\boldsymbol{x}, \boldsymbol{v} \in \mathbb{R}^{3 n}$, formed by permuting the components of $\boldsymbol{x}_{i}$ and $\boldsymbol{v}_{i}$, respectively, $\forall i$

$$
\boldsymbol{x}=\left[\boldsymbol{x}_{11}, \ldots, \boldsymbol{x}_{n 1}, \boldsymbol{x}_{12}, \ldots, \boldsymbol{x}_{n 2}, \boldsymbol{x}_{13}, \ldots, \boldsymbol{x}_{n 3}\right]^{\top}
$$

and $\boldsymbol{v}$ is constructed in a similar manner. There exists a permutation matrix, denoted by $P_{3 n} \in \mathbb{R}^{3 n \times 3 n}$, such that

$$
\boldsymbol{x}=P_{3 n}\left[\begin{array}{c}
\boldsymbol{x}_{1} \\
\boldsymbol{x}_{2} \\
\vdots \\
\boldsymbol{x}_{n}
\end{array}\right], \quad\left[\begin{array}{c}
\boldsymbol{x}_{1} \\
\boldsymbol{x}_{2} \\
\vdots \\
\boldsymbol{x}_{n}
\end{array}\right]=P_{3 n}^{-1} \boldsymbol{x} .
$$

Ignoring the pursuer-dependent terms, the flocking dynamics (4) can be written as

$$
\begin{aligned}
\dot{\boldsymbol{x}}= & \boldsymbol{v} \\
\dot{\boldsymbol{v}}= & -k_{a}\left(I_{3} \otimes D_{c} B_{c}^{\top}\right) \boldsymbol{v}+k_{g}\left(\boldsymbol{v}_{d} \otimes \mathbf{1}_{n}-\boldsymbol{v}\right) \\
& -k_{s}\left(I_{3} \otimes D_{c} Q(\boldsymbol{x}) B_{c}^{\top}\right) \boldsymbol{x}
\end{aligned}
$$

where $D_{c}(i, j)=-1$ if the $j$ th edge is incoming at $i$, and $D_{c}(i, j)=0$ otherwise. Here, $I_{3}$ the $3 \times 3$ identity matrix, while $\otimes$ denotes the Kronecker product. We can replace $D_{c}$ with $B_{c} / 2$ if the graph is undirected. The matrix $Q(\boldsymbol{x}) \in \mathbb{R}^{p \times p}$ is a diagonal matrix satisfying $Q(j, j)=1-R_{\text {safe }}^{3} /\left\|\boldsymbol{e}_{j}\right\|^{3}$.

In order to analyze the stability of the flock, we will make a change of coordinates: $\tilde{\boldsymbol{x}}_{i}(t)=\boldsymbol{x}_{i}(t)-\boldsymbol{v}_{d} t, \tilde{\boldsymbol{v}}_{i}(t)=\boldsymbol{v}_{i}(t)-$ $\boldsymbol{v}_{d}$. Since $Q(\boldsymbol{x})=Q(\tilde{\boldsymbol{x}})$, we write the flocking dynamics as follows:

$$
\begin{aligned}
& \dot{\tilde{\boldsymbol{x}}}=\tilde{\boldsymbol{v}} \\
& \dot{\tilde{\boldsymbol{v}}}=-k_{s}\left(I_{3} \otimes D_{c} Q(\tilde{\boldsymbol{x}}) B_{c}^{\top}\right) \tilde{\boldsymbol{x}}-k_{a}\left(I_{3} \otimes D_{c} B_{c}^{\top}\right) \tilde{\boldsymbol{v}}-k_{g} \tilde{\boldsymbol{v}} .
\end{aligned}
$$

This is a nonlinear, autonomous system whose equilibrium solutions are given by $\left[\tilde{\boldsymbol{x}}^{0}, \mathbf{1}_{n} \otimes 0_{3}\right]$, where $\tilde{\boldsymbol{x}}^{0}$ satisfies $\left(I_{3} \otimes\right.$
$\left.D_{c} Q\left(\tilde{\boldsymbol{x}}^{0}\right) B_{c}^{\top}\right) \tilde{\boldsymbol{x}}^{0}=0$. Given an equilibrium configuration $\tilde{\boldsymbol{x}}^{0}$, we define the family of equilibrium solutions obtained by rigidbody translation and rotation of the flock by

$$
\mathcal{S}\left(\tilde{\boldsymbol{x}}^{0}\right)=\left\{\tilde{\boldsymbol{x}} \mid \tilde{\boldsymbol{x}}=\mathbf{b} \otimes \mathbf{1}_{n}+\alpha P_{3 n}\left(I_{n} \otimes J\right) P_{3 n}^{-1} \tilde{\boldsymbol{x}}^{0}\right\}
$$

where $\mathbf{b} \in \mathbb{R}^{3}, J \in \mathbf{s o}(3)$, and $\alpha \in \mathbb{R}$.

Let $\delta$. denotes a small perturbation in the corresponding variable, and $\boldsymbol{e}_{i}^{0}$ is the steady-state $i$ th edge vector. Then, the linearization of (7) about an equilibrium point is given by

$$
\begin{aligned}
\delta \dot{\boldsymbol{x}}= & \delta \boldsymbol{v} \\
\delta \dot{\boldsymbol{v}}= & -\frac{3}{R^{2}}\left(I_{3} \otimes D_{c} \Delta\left(\boldsymbol{e}_{1}^{0} \delta \boldsymbol{e}_{1}, \ldots, \boldsymbol{e}_{p}^{0} \delta \boldsymbol{e}_{p}\right) B_{c}^{\top}\right) \tilde{\boldsymbol{x}}^{0} \\
& -\left(k_{a}\left(I_{3} \otimes D_{c} B_{c}^{\top}\right)+k_{g} I_{3 n}\right) \delta \boldsymbol{v} .
\end{aligned}
$$

These equations can be written compactly as $\left[\begin{array}{l}\delta \dot{\boldsymbol{x}} \\ \delta \dot{\boldsymbol{v}}\end{array}\right]=G\left[\begin{array}{l}\delta \boldsymbol{x} \\ \delta \boldsymbol{v}\end{array}\right]$, where the Jacobian $G$ is given by

$$
G=\left[\begin{array}{cc}
\mathbf{0}_{3 n} & I_{3 n} \\
-k_{s} A & -B
\end{array}\right]
$$

and

$$
\begin{aligned}
B & =k_{a}\left(I_{3} \otimes D_{c} B_{c}^{\top}\right)+k_{g} I_{3 n} \\
A \delta \boldsymbol{x} & =\frac{3}{R^{2}}\left(I_{3} \otimes D_{c} \Delta\left(\boldsymbol{e}_{1}^{0} \delta \boldsymbol{e}_{1}, \ldots, \boldsymbol{e}_{p}^{0 \top} \delta \boldsymbol{e}_{p}\right) B_{c}^{\top}\right) \tilde{\boldsymbol{x}}^{0} .
\end{aligned}
$$

\section{B. Spectral Properties of $A$ and $G$}

Lemma 1: The null space of $A$ defined in (10) is a superset of eigenvectors, which correspond to a rigid body translation and rotation of the flock; i.e.,

$$
\begin{aligned}
& \mathcal{N}(A) \supseteq \mathcal{N}_{u} \triangleq \operatorname{span}\left([1,0,0]^{\top} \otimes \mathbf{1}_{n},[0,1,0]^{\top} \otimes \mathbf{1}_{n},\right. \\
& {[0,0,1]^{\top} \otimes \mathbf{1}_{n}, P_{3 n}\left(I_{n} \otimes J_{1}\right) P_{3 n}^{-1} \tilde{\boldsymbol{x}}^{0}, P_{3 n}\left(I_{n} \otimes J_{2}\right) P_{3 n}^{-1} \tilde{\boldsymbol{x}}^{0},} \\
& \left.P_{3 n}\left(I_{n} \otimes J_{3}\right) P_{3 n}^{-1} \tilde{\boldsymbol{x}}^{0}\right)
\end{aligned}
$$

where $J_{1}, J_{2}, J_{3}$ form a basis for so(3).

Proof: It is evident from (10) that $\mathcal{N}(A)$ is a superset of the vectors $\delta \boldsymbol{x}$, which satisfy $\left(I_{n} \otimes B_{c}^{\top}\right) \delta \boldsymbol{x}=$ 0 or $\boldsymbol{e}_{i}^{0} \delta \boldsymbol{e}_{i}=0 \forall i=\{1, \ldots, p\}$. The former condition implies that $\delta \boldsymbol{x}=k_{1}[1,0,0]^{\top} \otimes \mathbf{1}_{n}+k_{2}[0,1,0]^{\top} \otimes \mathbf{1}_{n}+$ $k_{3}[0,0,1]^{\top} \otimes \mathbf{1}_{n}$, where $k_{1}, k_{2}$, and $k_{3}$ are arbitrary constants. The latter condition is satisfied by $\delta \boldsymbol{x}_{i}=\sum_{j=1}^{3} k_{j}^{\prime} J_{j} \boldsymbol{x}_{i}$ for all constants $k_{j}^{\prime}$.

Assumption 1: The matrix $A$ has exactly six eigenvalues, which are equal to 0 . Therefore, $\mathcal{N}_{u}(A)=\mathcal{N}(A)$.

Assumption 1 is satisfied by connected undirected graphs [27] and by strongly connected and balanced digraphs [16].

Assumption 2: The Jacobian $G$ has the following properties.

1) All eigenvalues of $G$ have nonpositive real parts.

2) The algebraic and geometric multipicities of the zero eigenvalue are equal to each other.

3) The null space $\mathcal{N}(G)=\left[\mathbf{n}^{\top}, \mathbf{0}_{1 \times 3 n}\right]^{\top}$, where $\mathbf{n} \in$ $\mathcal{N}(A)=\mathcal{N}_{u}(A)$.

The reader should note that Assumption 2 always holds for an undirected graph satisfying Assumption 1 [27]. 


\section{Stability Analysis}

In this section, we use the center manifold theorem [42, Th. 1] to show that trajectories which start away from $\mathcal{S}\left(\tilde{\boldsymbol{x}}^{0}\right)$, at least locally, converge to $\mathcal{S}\left(\tilde{\boldsymbol{x}}^{0}\right)$ at an exponential rate. The proof proceeds in two steps. First, we show that there exists a center manifold when Assumption 2 is satisfied. We note that the set $\mathcal{S}\left(\tilde{\boldsymbol{x}}^{0}\right)$ is also a center manifold. In the second step, we use the property that center manifolds are arbitrarily close to each other to complete the proof.

Lemma 2: Suppose that the flocking dynamics satisfy Assumption 2. Then, for every steady state $\tilde{\boldsymbol{x}}^{0}$, there exists an invariant manifold $\mathcal{E}^{c} \triangleq \mathcal{E}^{c}\left(\tilde{\boldsymbol{x}}^{0}\right)$, such that any trajectory starting around $\mathcal{E}^{c}$ converges at an exponential rate to $\mathcal{E}^{c}$.

Proof: From Assumption 2, we know that the Jacobian $G$, evaluated at $\tilde{\boldsymbol{x}}^{0}$, has six zero eigenvalues and the other eigenvalues are negative. Thus, it follows from the center manifold theorem [42, Th. 1] that there exists a 6-D center manifold $\mathcal{E}^{c}$ such that trajectories starting outside $\mathcal{E}^{c}$ converge at an exponential rate to $\mathcal{E}^{c}$.

Theorem 1: The set $\mathcal{S}\left(\tilde{\boldsymbol{x}}^{0}\right)$ is exponentially stable.

Proof: We note that $\mathcal{S}\left(\tilde{\boldsymbol{x}}^{0}\right)$ is itself a center manifold of the dynamics. From Lemma 2, we know that trajectories starting in a neighborhood of $\boldsymbol{x}^{0}$ converge at an exponential rate to the center manifold $\mathcal{E}^{c}\left(\tilde{\boldsymbol{x}}^{0}\right)$. We recall that any two center manifolds about an equilibrium point $\tilde{\boldsymbol{x}}^{0}$ differ by transcendentally small terms, and every equilibrium point in the vicinity of $\tilde{\boldsymbol{x}}^{0}$ lies on every center manifold $\mathcal{E}^{c}\left(\tilde{\boldsymbol{x}}^{0}\right)$ [42, Sec. 4.6]. Thus, a trajectory starting in a neighborhood of $\tilde{\boldsymbol{x}}^{0}$ converges exponentially fast to $\mathcal{S}\left(\tilde{\boldsymbol{x}}^{0}\right)$.

The discussion above shows that $\mathcal{S}\left(\tilde{\boldsymbol{x}}^{0}\right)$ is exponentially stable, and therefore, robust to small perturbations. In the context of herding, this implies that, for every direction in relation to the flock (or its center), there exists a nontrivial set of pursuer positions for which the underlying graph of the flock is preserved. In the next section, we present analytical formulae for estimating a set of permissible pursuer positions based purely on local topological considerations.

In addition to stability, for the purpose of herding, it is useful to have the property of time-scale separation between the synchronization of the flocking dynamics to $\mathcal{S}\left(\tilde{\boldsymbol{x}}^{0}\right)$ on the one hand, and the translational dynamics of the center of gravity of the flock (whose time constant, as shown in Section V, is $1 / k_{g}$ ) on the other [21], [22]. This can be achieved by a suitable choice of $k_{s}$ and $k_{a}$ for a given graph topology.

\section{Analytical Estimation of the Permissible Set of PURSUER POSITIONS}

The objective of this section is to derive approximations for the approach distance between a pursuer and the flock, based purely on the local interaction between the pursuer and the birds in a flock. This estimate supersedes the distance $R_{\text {agg }}$ in Fig. 2 from the point of view of preserving the local topology of the flock, and is used in Section $\mathrm{V}$ to determine the feasible positions for the pursuer in relation to the flock.

The influence exerted by the pursuer can cause one of the two effects in extreme circumstances. In one case, the pursuer

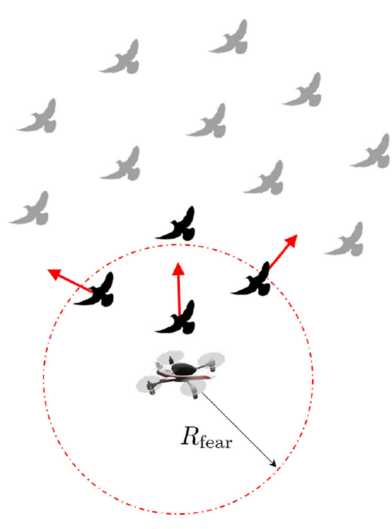

(a)

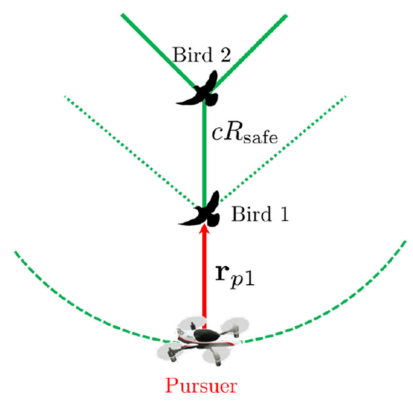

(b)
Fig. 4. Typical subgraph in a dense flock, and a subgraph with two birds collinear with the pursuer. Note $c R_{\text {safe }}<R_{\text {safe }}$. (a) Dense flock. (b) Local neighborhood.

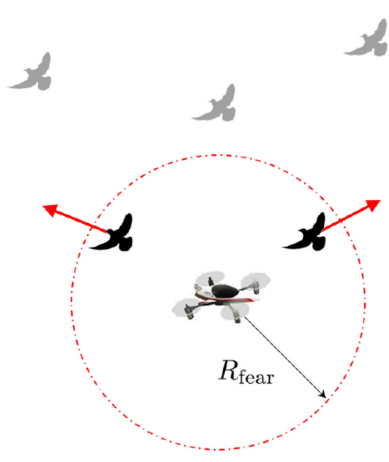

(a)



(b)
Fig. 5. Model with a single edge and the pursuer. The pursuer approaches along the perpendicular bisector of the edge and $\mathbf{r}_{p 1}=\mathbf{r}_{p 2}$. (a) Sparse flock. (b) Local neighborhood.

can push a bird to within an unacceptable distance of its neighbor. This scenario is likely in dense flocks, as shown in Fig. 4. In the second case, the pursuer can cause the link between two neighbors to be broken by pushing them beyond their communication distance $R_{\text {com }}$. This scenario, illustrated in Fig. 5, is more plausible in sparse flocks. We write the minimum permissible distance between two birds as $c R_{\text {safe }}$, where $c<1$ is a constant that depends on the species of birds and the pursuing UAV.

\section{A. Maintaining Minimum Allowable Distance Between Neighbors}

Consider the situation wherein a bird on the boundary of the flock (labeled "1") is engaged by the pursuer. The bird tries to move away radially from the pursuer, and into the flock. In the worst case, the bird's evasive path points in the direction of a neighboring bird ("2") and there is force from other neighboring birds pulling 1 away from 2. This is depicted in Fig. 4.

A conservative estimate for the minimum approach distance between the pursuer and the bird 1 is found from Fig. 4(b). This is a repelling, "outward-pointing" (with respect to the flock) force on 1 due to 2 is countered by the inward-pointing force on 1 
arising as a consequence of repulsion by the pursuer. Balancing these two forces when the distance between 1 and 2 is $c R_{\text {safe }}$ (thereby, ensuring that the minimum approach distance is no less than $R_{\text {safe }}$ ) gives the minimum approach distance $\left\|\mathbf{r}_{p 1}\right\|_{\text {min }}$

$$
\begin{aligned}
\frac{k_{p}}{\left\|\mathbf{r}_{p 1}\right\|_{\text {min }}^{2}} & =k_{s}\left|c R_{\text {safe }}\left(1-\frac{R_{\text {safe }}^{3}}{\left(c R_{\text {safe }}\right)^{3}}\right)\right| \\
& \Rightarrow\left\|\mathbf{r}_{p 1}\right\|_{\text {min }}=\sqrt{\frac{c^{2} k_{p}}{\left(1-c^{3}\right) k_{s} R_{\text {safe }}}} .
\end{aligned}
$$

\section{B. Preserving Communication Between Neighboring Birds}

In sparse flocks, it is a commonplace to find a linear topology, involving two or three agents forming an angle or a straight line with no other common neighbors. Consider a single edge, as shown in Fig. 5, involving just two birds, and assume that the pursuer approaches along the perpendicular bisector of the edge. If $R_{\text {com }}$ is the maximum permissible distance between two boundary agents for the underlying graph to be preserved, we get the following condition for the minimum permissible $\left\|\mathbf{r}_{p 1}\right\|$

$$
\begin{gathered}
\frac{k_{p}}{\left\|\mathbf{r}_{p 1}\right\|^{2}} \times \frac{R_{\mathrm{com}}}{2\left\|\mathbf{r}_{p 1}\right\|}=k_{s}\left(1-\frac{R_{\mathrm{safe}}^{3}}{R_{\mathrm{com}}^{3}}\right) R_{\mathrm{com}} \\
\Rightarrow\left\|\mathbf{r}_{p 1}\right\|_{\min }=\left(\frac{k_{p}}{2 k_{s}\left(1-\frac{R_{\mathrm{safe}}^{3}}{R_{\mathrm{com}}^{3}}\right)}\right)^{1 / 3} .
\end{gathered}
$$

We will provide a numerical example for $\left\|\mathbf{r}_{p 1}\right\|_{\min }$ in Section VIII. In Section V, the estimates for $\left\|\mathbf{r}_{p 1}\right\|_{\text {min }}$ are used as part of the motion planning strategy for the pursuer.

Definition 1: We define a set $\mathcal{X}_{p}$ of permissible pursuer positions in relation to the flock

$$
\mathcal{X}_{p}=\left\{\boldsymbol{x}_{p} \mid R_{\min } \leq \min _{i \in \mathcal{V}}\left\|\boldsymbol{x}_{p}-\boldsymbol{x}_{i}\right\| \leq R_{\text {fear }}\right\}
$$

where $R_{\mathrm{min}}$ is the maximum of $R_{\mathrm{agg}}$ and either (11) or (12) depending on the topology of the flock.

\section{V. $m$-WAYPOINT HERDING AlgORITHM}

In Sections III and IV, we established conditions for stability and robustness of the flock, and derived approximations for the approach distance between the flock and the pursuer. In this section, we solve the problem of herding, described in Section II-C, by modeling the ensemble behavior of the flock. We first derive a set of objectives that the pursuer's trajectory, $\boldsymbol{x}_{p}(t)$, must satisfy in order to herd the flock successfully. The $m$-waypoint algorithm is obtained as an approximate, but efficient, solution to the problem of ensuring that $\boldsymbol{x}_{p}(t)$ meets these objectives.

\section{A. Formulation of the Herding Problem}

The coordinates of the flock's centroid and its velocity are given by $\boldsymbol{x}_{c g}=\frac{1}{n} \sum_{i=1}^{n} \boldsymbol{x}_{i}, \boldsymbol{v}_{c g}=\frac{1}{n} \sum_{i=1}^{n} \boldsymbol{v}_{i}$. Using (4) and
(5), we get

$$
\begin{aligned}
\dot{\boldsymbol{x}}_{c g} & =\boldsymbol{v}_{c g} \\
\dot{\boldsymbol{v}}_{c g} & =k_{g}\left(\boldsymbol{v}_{d}-\boldsymbol{v}_{c g}\right)+\mathbf{F}_{p}\left(\boldsymbol{x}_{p}\right)+\boldsymbol{f}(\boldsymbol{x}, \boldsymbol{v}) \\
\mathbf{F}_{p}\left(\boldsymbol{x}_{p}\right) & \triangleq \frac{k_{p}}{n} \sum_{i \in \mathcal{N}_{p}} \boldsymbol{H}\left(\mathbf{r}_{p i}\right) \\
\dot{\boldsymbol{x}}_{p} & =\boldsymbol{u}_{p}
\end{aligned}
$$

where $\mathcal{N}_{p}$ denotes the subset of the flock that lies within $R_{\text {fear }}$ of the pursuer; the pursuer's velocity, $\boldsymbol{u}_{p}$, is a control variable for the herding algorithm. The term $\boldsymbol{f}(\boldsymbol{x}, \boldsymbol{v})$ is zero when the graph is undirected (since $\mathbf{1}_{n}^{\top} B_{c}=0$ ) or if it has synchronized to a steady state configuration from Section III. This would occur naturally when the flock synchronizes on a faster time scale than the dynamics of its CG [21], [22]. Since we need the flock to point in the direction of the herding target point, it suffices to ensure that its velocity normal to an axis pointing towards $\boldsymbol{x}_{\mathrm{div}}$ is driven to zero. We define

$$
\mathbf{r}_{\mathrm{dc}}(t) \triangleq \boldsymbol{x}_{\mathrm{div}}(t)-\boldsymbol{x}_{c g}(t), \quad \mathbf{q} \triangleq \mathbf{r}_{\mathrm{dc}} \times \boldsymbol{v}_{c g}
$$

Recall, from Section II-C, that $\hat{\mathbf{k}}^{\top} \boldsymbol{x}_{\mathrm{div}}(t)=\hat{\mathbf{k}}^{\top} \boldsymbol{x}_{c g}(t)=h(t)$ (the altitude of the flock). Since $\boldsymbol{x}_{\text {div }}(t)$ lies on a fixed vertical line for all $t$, we also have that $\hat{\mathbf{k}} \times \dot{\boldsymbol{x}}_{\text {div }}(t)=0$. Since $\dot{\mathbf{r}}_{\mathrm{dc}}(t)=\dot{\boldsymbol{x}}_{\mathrm{div}}(t)-\boldsymbol{v}_{c g}(t)$, we get the following expression for the dynamics of $\mathbf{q}$ :

$$
\begin{aligned}
\dot{\mathbf{q}}= & \mathbf{r}_{\mathrm{dc}} \times \dot{\boldsymbol{v}}_{c g}+\dot{\boldsymbol{x}}_{\mathrm{div}} \times \boldsymbol{v}_{c g} \\
= & -k_{g} \mathbf{q}+k_{g}\left(\mathbf{r}_{\mathrm{dc}} \times \boldsymbol{v}_{d}\right)+\mathbf{r}_{\mathrm{dc}} \times \mathbf{F}_{p}\left(\boldsymbol{x}_{p}\right)+\mathbf{r}_{\mathrm{dc}} \times \boldsymbol{f}(\boldsymbol{x}, \boldsymbol{v}) \\
& +\dot{\boldsymbol{x}}_{\mathrm{div}} \times \boldsymbol{v}_{c g} .
\end{aligned}
$$

Next, we define $q_{k} \triangleq \hat{\mathbf{k}}^{\top} \mathbf{q}$. Note that $\hat{\mathbf{k}}^{\top}\left(\dot{\boldsymbol{x}}_{\mathrm{div}} \times \boldsymbol{v}_{c g}\right)=$ $\boldsymbol{v}_{c g}^{\top}\left(\hat{\mathbf{k}} \times \dot{\boldsymbol{x}}_{\text {div }}\right)=0$. Thus, the dynamics of $q_{k}$ are given by

$$
\dot{q}_{k}+k_{g} q_{k}=\hat{\mathbf{k}}^{\top}\left(\mathbf{r}_{\mathrm{dc}} \times\left(k_{g} \boldsymbol{v}_{d}+\boldsymbol{f}(\boldsymbol{x}, \boldsymbol{v})\right)+\mathbf{r}_{\mathrm{dc}} \times \mathbf{F}_{p}\left(\boldsymbol{x}_{p}\right)\right) .
$$

We denote the amount of deviation that can be produced by placing the pursuer at $\boldsymbol{x}_{p}$ using the right hand-side of (16):

$$
\rho\left(\boldsymbol{x}_{p}\right) \triangleq \hat{\mathbf{k}}^{\top}\left(\mathbf{r}_{\mathrm{dc}} \times\left(k_{g} \boldsymbol{v}_{d}+\boldsymbol{f}(\boldsymbol{x}, \boldsymbol{v})\right)+\mathbf{r}_{\mathrm{dc}} \times \mathbf{F}_{p}\left(\boldsymbol{x}_{p}\right)\right)
$$

If $\rho\left(\boldsymbol{x}_{p}\right)=0$ for all $t$, we deduce from (16) that $q_{k} \rightarrow 0$ and the $\mathrm{CG}$ of the flock moves in a straight line (solid line in Fig. 6) towards $\boldsymbol{x}_{\text {div }}$ (from the definition of $q_{k}$ ). We also note that the distance and the time required for herding could be reduced, as compared to moving the flock along the solid line, by pushing it rapidly towards the dashed line passing through $x_{\text {div }}$ in Fig. 6. The dashed line is parallel to $\boldsymbol{v}_{d}$, and once the flock's CG reaches it, the herding algorithm can safely terminate. This requires that $q_{k} \geq 0$ uniformly (or $q_{k} \leq 0$ uniformly, depending on the choice of $\boldsymbol{x}_{\text {div }}$ ) during the course of herding. Without loss of generality, we assume that $\boldsymbol{x}_{\mathrm{div}}$ is chosen, $\operatorname{such}$ that $\operatorname{sign}\left(\hat{\mathbf{k}}^{\top}\left(\boldsymbol{v}_{d} \times \mathbf{r}_{\mathrm{dc}}\right)\right)>0$ while commencing herding and set the control objective to maximizing $\rho\left(\boldsymbol{x}_{p}\right) \geq 0$ while $\operatorname{sign}\left(\hat{\mathbf{k}}^{\top}\left(\boldsymbol{v}_{d} \times \mathbf{r}_{\mathrm{dc}}\right)\right)>0$ (see Fig. 6). 


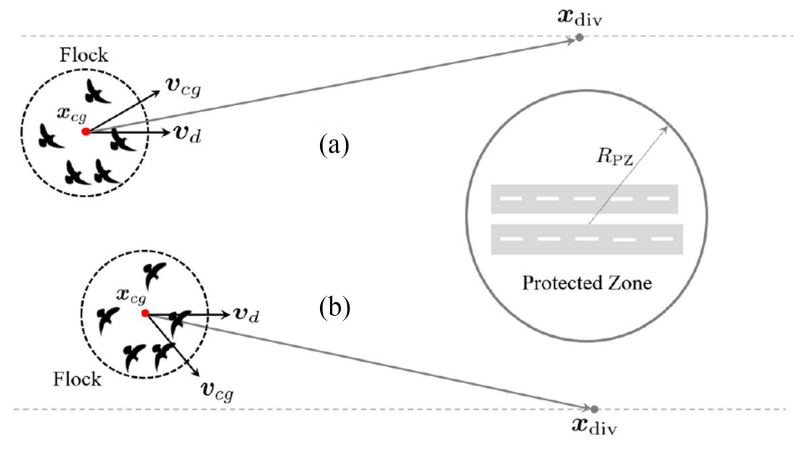

Fig. 6. Two canonical scenarios, with different signs of $q_{k}$, that correspond to the flock reaching the dashed line faster than by moving along the solid line to $\boldsymbol{x}_{\text {div }}$ (which corresponds to $q_{k}=0$ ). Once on the dashed line, which is parallel to $\boldsymbol{v}_{d}$, the flock is guaranteed to stay outside PZ. (a) $q_{k}>0$, (b) $q_{k}<0$.

\section{B. Determination of $m$ Waypoints}

An ideal solution to the aforementioned control problem is to compute the trajectory of $\boldsymbol{x}_{p}(t)$ which maximizes $\rho\left(\boldsymbol{x}_{p}\right)$, and get the pursuer to track it. It is well known that a flock tends to deform into a concave shape locally under persistent pressure from a pursuer, which is known to dent the effectiveness of the pursuit [29]. Furthermore, over stressing one or more birds continuously over an extended period of time carries the risk of the distressed birds attempting aggressive evasive maneuvers and fragmenting the flock. To avoid these problems, we use a sub optimal approach wherein the flock is engaged through different waypoints in a given time frame.

The waypoints are chosen by sampling the set $\mathcal{X}_{p}$ from Definition 1. Sampling is preferred to statically defined waypoints because it allows the algorithm to be agnostic to the exact geometry of the flock. This is useful when the flock is not necessarily best represented as a convex shape, for instance, star-shaped flocks and flocks with a curvilinear geometry. We formally identify the set of $m$ waypoints as follows.

Definition 2: The set $\mathcal{X}_{p}^{m}$ is defined by construction. The waypoint selection algorithm, described in Algorithm 2, samples $\mathcal{X}_{p}$ uniformly. Next, up to $m$ waypoints with the highest deviation are identified such that no two waypoints are within a prescribed distance, denoted $\delta_{w}$, of each other. These waypoints constitute the set $\mathcal{X}_{p}^{m}$.

Fig. 7 shows an example of the set $\mathcal{X}_{p}^{m}$ for an arbitrarily chosen flock and deterministically chosen sample points. It illustrates that even for a convex shaped flock, the set $\mathcal{X}_{p}^{m}$ need not be connected. This is one of the reasons why we use random sampling to construct it.

\section{Motion Planning for the Pursuer}

The motion planner solves for the pursuer's velocity $\boldsymbol{u}_{p}(t)$ by commanding one of two motions, as follows.

1) FLY: the pursuer takes the fastest path to the commanded node. Collision avoidance is achieved using artificial potential fields, an alternative to which is the real time, online motion planner based on [43].

2) ENGAGE: using a virtual leader-based approach, the pursuer commands $\boldsymbol{u}_{p}(t)$ to maintain its position at the chosen

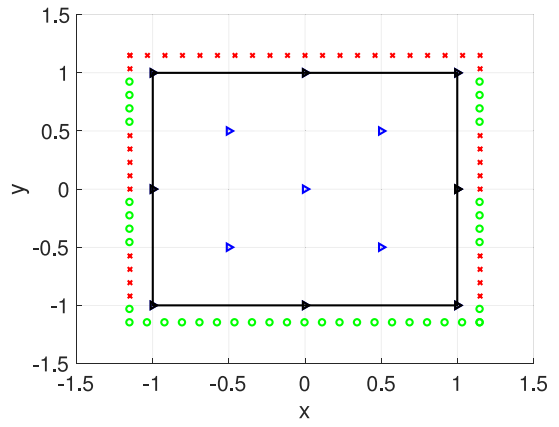

Fig. 7. For an example scenario where the force is to be applied along the $+y$ direction, the candidate waypoints in $\mathcal{X}_{p}$, defined in (13), are grouped into two categories. Points marked in green circles satisfy $\rho\left(\boldsymbol{x}_{p}\right)>0$ and contain the set $\mathcal{X}_{p}^{m}$ (see Definition 2). Points marked by red $\times$ do not satisfy $\rho\left(\boldsymbol{x}_{p}\right)>0$. Here, blue and black triangles denote birds in the interior of the flock and its convex hull, respectively.

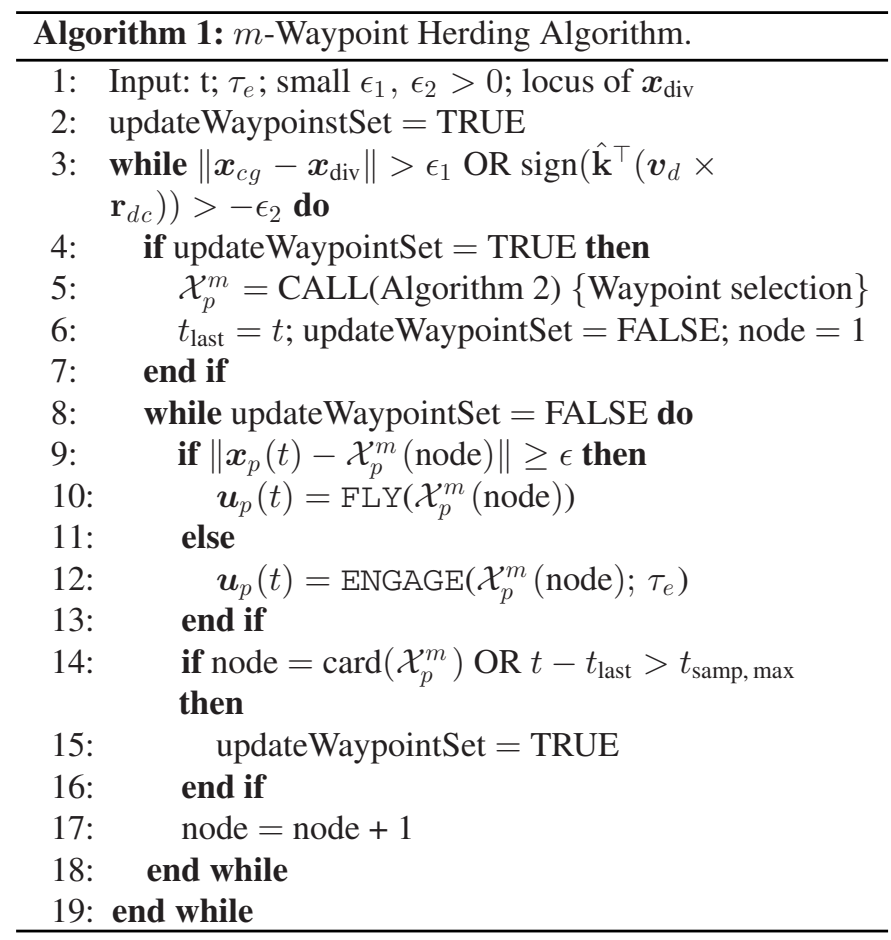

waypoint in relation to the flock's centroid for a predetermined duration $\tau_{e}$. The engagement is terminated if the distance between two neighboring birds in $\mathcal{N}_{p}$ approaches the communication radius, $R_{\text {com }}$.

\section{ANALYSIS OF THE HERDING AlgORITHM}

In this section, we derive a condition for successful herding, in the form of the minimum allowable distance from the PZ at which the herding must begin. If the herding commences beyond this distance, it will almost surely succeed. We assume that $\boldsymbol{f}(\boldsymbol{x}, \boldsymbol{v})=0$, i.e., the underlying graph is undirected or the flock has synchronized to a steady state.

We start by ignoring the dynamics of the pursuer, and recall that the control objective is to maximize $\rho\left(\boldsymbol{x}_{p}\right) \geq 0$. We consider the conservative case $\rho\left(\boldsymbol{x}_{p}\right)=0$ for all $t$ during the course of 


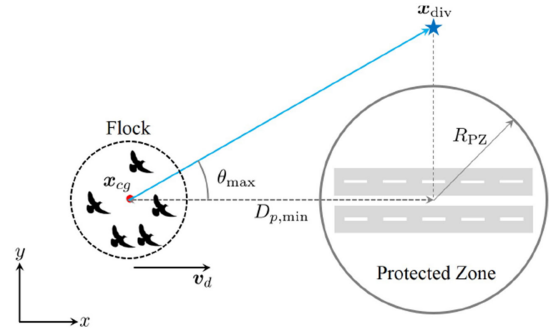

Fig. 8. One half of the cone outside which the herding must start; the other half is the mirror image of the shown half.

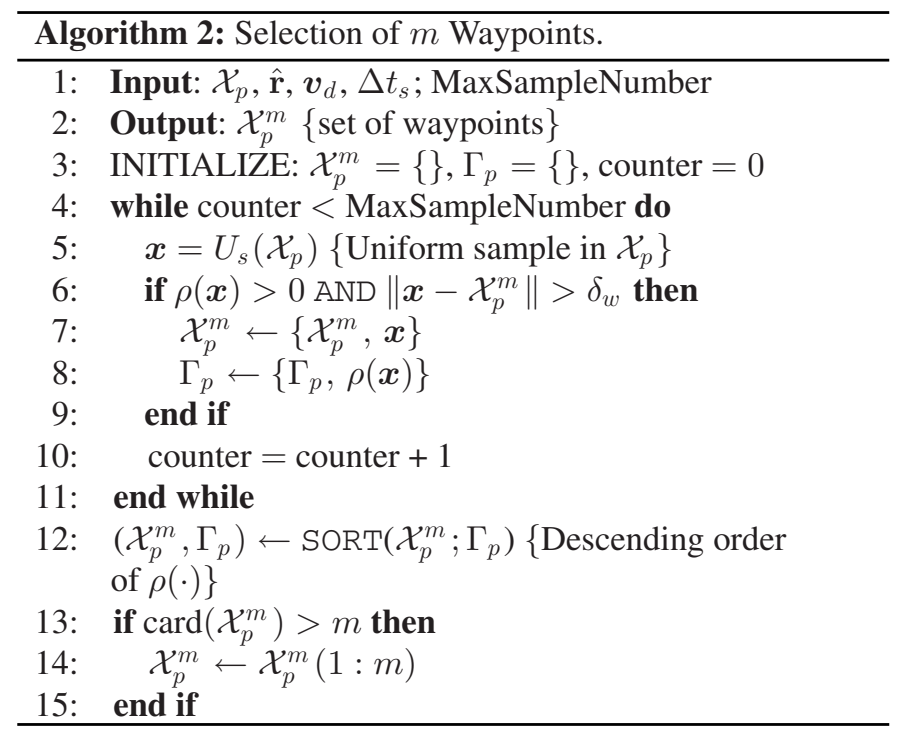

herding. This case is limiting in that the distance at which the herding needs to be commenced can be lesser when larger values of $\rho\left(\boldsymbol{x}_{p}\right)$ are attainable (see Section V-A).

In order to achieve $\rho\left(\boldsymbol{x}_{p}\right)=0$, we set $\boldsymbol{x}_{p}=\boldsymbol{x}_{p}^{*}$ where $\mathbf{F}_{p}\left(\boldsymbol{x}_{p}^{*}\right)=-k_{g}\left(\hat{\mathbf{k}}^{\top}\left(\hat{\mathbf{r}}_{\mathrm{dc}} \times \boldsymbol{v}_{d}\right)\right)\left(\hat{\mathbf{k}} \times \hat{\mathbf{r}}_{\mathrm{dc}}\right)$. Since $\hat{\mathbf{r}}_{\mathrm{dc}}=$ $\mathbf{r}_{\mathrm{dc}} /\left\|\mathbf{r}_{\mathrm{dc}}\right\|$, and $\boldsymbol{v}_{d}$ lie in the horizontal plane, it follows that $\left\|\mathbf{F}_{p}\right\|=k_{g}\left\|\hat{\mathbf{r}}_{\mathrm{dc}} \times \boldsymbol{v}_{d}\right\|$. Let $\rho_{F}$ denote the upper bound on $\left\|\mathbf{F}_{p}\right\|$, which is known from the analysis in Section IV. It is clear then that the flock should be made to satisfy $\left\|\hat{\mathbf{r}}_{\mathrm{dc}} \times \boldsymbol{v}_{d}\right\|<$ $\rho_{F} / k_{g} \forall t$, which, in turn, means that the flock must be kept outside the cone shown in Fig. 8. This is an important insight: it shows that the herding is successful only if it begins when the horizontal distance between the center of the flock and the center of $\mathrm{PZ}$ is greater than or equal to

$$
D_{p, \text { min }} \triangleq \frac{R_{\mathrm{div}}}{\tan \theta_{\max }}, \theta_{\max }=\sin ^{-1}\left(\frac{\rho_{F}}{k_{g}\left\|\boldsymbol{v}_{d}\right\|}\right)
$$

where $R_{\text {div }}$ was defined in Section II-C.

While the solution $\boldsymbol{x}_{p}^{*}$ appears to solve the problem in principle, there is the possibility that the trajectory $\boldsymbol{x}_{p}^{*}$ may not be feasible. Furthermore, the motion planning algorithm adopted here requires that the pursuer engage with an entire "front" of the flock rather than specific individual birds. In particular, it means that the engagement between the pursuer and the flock takes

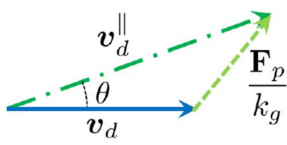

(a)

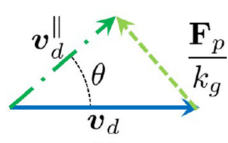

(b)
Fig. 9. Vector diagram showing $\boldsymbol{v}_{d}^{\|}$and $\theta$ for push (a) from the aft and (b) from the front, respectively.

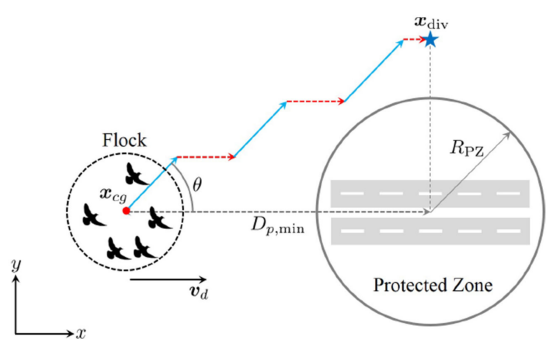

Fig. 10. Refining the estimate of $D_{p, \text { min }}$ assuming a bimodal herding model. The solid blue segments denote the motion of the flock when the pursuer engages it actively; the dashed red segments denote the worst-case motion in the absence of any pursuer engagement.

place in pulses. We refine the necessary condition to account for this pulsed interaction.

Let $\tau_{e}$ denote the duration of any given engagement between the pursuer and the flock, and let $\tau_{f}$ denote the time taken by the bird to fly between two waypoints. During the time $\tau_{f}$, the flock receives no external stimulus and its velocity tends to align to $\boldsymbol{v}_{d}$. Assuming that an engagement begins at time $t_{0}$, the dynamics of the flock in the time interval $\left[t_{0}, t_{0}+\tau_{e}+\tau_{f}\right]$ can be described via the switching dynamics

$$
\dot{\boldsymbol{v}}_{c g}+k_{g} \boldsymbol{v}_{c g}= \begin{cases}k_{g} \boldsymbol{v}_{d}^{\|}, & t \in\left[t_{0}, t_{0}+\tau_{e}\right) \\ k_{g} \boldsymbol{v}_{d}, & t \in\left[t_{0}+\tau_{e}, t_{0}+\tau_{e}+\tau_{f}\right]\end{cases}
$$

where $\boldsymbol{v}_{d}^{\|}=\boldsymbol{v}_{d}+\mathbf{F}_{p} / k_{g}$ (see Fig. 9). When $\boldsymbol{x}_{p}=\boldsymbol{x}_{p}^{*}, \boldsymbol{v}_{d}^{\|}$is along $\mathbf{r}_{\mathrm{dc}}$ at every instant in time, which is why the superscript |l is used here. We will assume that the flock switches instantaneously between two states. During the engagement phase, the flock moves at an angle $\theta$ to the original direction coinciding with $\boldsymbol{v}_{d}$. When the pursuer does not engage the flock, it moves along $\boldsymbol{v}_{d}$. This is depicted in Fig. 10.

The terms $\left\|\boldsymbol{v}_{d}^{\|}\right\|$and $\theta$ should ideally be estimated empirically, as in Section VII-A, since the precise interaction between the flock and the pursuer is highly case-specific. The magnitude of $v_{d}^{\|}$depends on whether the flock is driven from the front or from the rear. Since we intend for the net motion to be perpendicular to $\boldsymbol{v}_{d}$, it is reasonable to assume that the average force also acts perpendicular to $\boldsymbol{v}_{d}$. Thus, we get

$$
\left\|\boldsymbol{v}_{d}^{\|}\right\|=\sqrt{\left\|\boldsymbol{v}_{d}\right\|^{2}+\frac{\left\|\mathbf{F}_{p}\right\|^{2}}{k_{g}^{2}}}, \theta=\cos ^{-1}\left(\left\|\boldsymbol{v}_{d}\right\| /\left\|\boldsymbol{v}_{d}^{\|}\right\|\right) .
$$

We now state the main result of this section. 
Theorem 2: Given the approximate values of $\left\|\boldsymbol{v}_{d}^{\|}\right\|$and $\theta$, the refined value of $D_{p, \text { min }}$ is given by

$$
D_{p, \text { min }}=R_{\mathrm{div}} \cot \theta\left(1+\frac{\tau_{f}}{\tau_{e}}\right) .
$$

Proof: Let $n_{s}$ denote the number of engagement pulses. From Fig. 10, it is apparent that we can have at most $n_{s}$ pulses wherein the flock is not engaged with the pursuer. During each engagement pulse, referring to the geometric convention of Fig. 10, the flock shifts through a distance $\left\|\boldsymbol{v}_{d}^{\|}\right\| \tau_{e} \sin \theta$. Thus,

$$
n_{s}=\frac{R_{\mathrm{div}}}{\left\|\boldsymbol{v}_{d}^{\|}\right\| \tau_{e} \sin \theta} .
$$

Furthermore, during each engagement phase, the flock translates horizontally through a distance $\left\|\boldsymbol{v}_{d}^{\|}\right\| \tau_{e} \cos \theta$. During the nonengagement phase, the horizontal translation is at most $\left\|\boldsymbol{v}_{d}\right\| \tau_{f}$. Thus, the minimum horizontal translation is given by

$$
\begin{aligned}
D_{p, \text { min }} & =n_{s}\left(\left\|\boldsymbol{v}_{d}\right\| \tau_{f}+\left\|\boldsymbol{v}_{d}^{\|}\right\| \tau_{e} \cos \theta\right) \\
& =R_{\operatorname{div}}\left(\frac{\left\|\boldsymbol{v}_{d}\right\|}{\left\|\boldsymbol{v}_{d}^{\|}\right\|} \frac{\tau_{f}}{\tau_{e}} \frac{1}{\sin \theta}+\cot \theta\right)
\end{aligned}
$$

Setting $\cos \theta=\left\|\boldsymbol{v}_{d}\right\| /\left\|\boldsymbol{v}_{d}^{\|}\right\|$yields $D_{p, \text { min }}=R_{\text {div }} \cot \theta(1+$ $\left.\frac{\tau_{f}}{\tau_{e}}\right)$. Notice that we recover (18) if we set $\tau_{f}=0$ and $\theta=\theta_{\max }$. This completes the proof.

Remark 1: We note two extreme cases that give further insight on the variation of $D_{p, \min }$ with the size of the flock. When $n$ is small, it is reasonable to expect that the pursuer simultaneously affects all members of the flock. In such cases, $\theta$, and subsequently, $D_{p, \text { min }}$ are independent of $n$ (the size of the flock). When $n$ is large, it is reasonable to assume that the pursuer engages a fixed number of boundary agents, and this number is also independent of $n$. Furthermore, we expect that $\theta$ is small, so that $\cot \theta \approx 1 / \theta$, and $\theta \approx\left\|\mathbf{F}_{p}\right\| /\left(k_{g}\left\|\boldsymbol{v}_{d}\right\|\right)$. Since the number of birds engaged by the pursuer is a constant, $\left\|\mathbf{F}_{p}\right\| \propto k_{f} / n$, where $k_{f}$ is a constant. Assuming that $\tau_{f} / \tau_{e}$ is not a function of $n$, it follows that $D_{p, \min } \propto n$; i.e., the minimum distance grows linearly with the flock size for large flocks.

Remark 2: In [7], we considered several cases wherein the total number of waypoints was fixed; for each case, a different fraction of waypoints was used in a manner consistent with this paper, while the rest were used for pushing the flock from the rear. This is analogous, in the framework of this paper, to changing the value of $m$. Therefore, we do not analyze the effect of changing $m$ in this sequel. We note, however, that the choice of the number of waypoints depends on the inherent cohesiveness of the flock. A larger number of waypoints may be needed to ensure containment in a loosely bound flock.

In Section VII, we will demonstrate how (19) can be used to extract a set of flocking model parameters from experimental data. The model for the minimum distance will be revisited in Section VIII (numerical simulations), with the objective of deriving an empirical relationship between the minimum distance and the size of the flock.

\section{EXPERIMENTS ON A LIVE FLOCK OF BIRDS}

In this section, we describe the results of experiments carried out on live birds in Daejeon, South Korea. The objective of the experiments was to validate the flocking model described earlier, and estimate the values of the parameters in the flocking model. The experiments were carried out in two rounds on two families of birds.

The experiments involved flying a quadrotor drone in the vicinity of flocks. Two rounds of experiments were conducted during two different seasons - the first involved egrets, while the second involved loons. For this study, two drones were deployed: One drone performed various types of maneuvers around the flocks as a pursuer (herding drone), while a surveillance drone hovered at a high altitude with a camera pointing directly down to the ground for recording the trajectories of the pursuer drone and the birds. Videos recorded during the experiments are available as part of the supplementary material, accompanying the paper.

\section{A. Experiments on Egrets}

Egrets are migratory birds, and at the time of conducting the experiments, they had settled in their nesting grounds in the vicinity of the KAIST campus. The birds make frequent visits to a hunting area nearby, and a large number of egrets are found to return to their nests at sunset. During this time, we attempted to fly the herding drone in various directions with respect to the flock.

In Figs. 11 and 12, two cases are closely studied. In Fig. 11, the drone approaches the flock horizontally at a sufficient distance. Initially, the birds fly along the $-x$ direction and the drone approaches from the left with about $30 \mathrm{~m}$ of clearance. As the birds discover the drone, they divert from their original paths and fly at a $45^{\circ}$ angle to their right. Meanwhile, the drone moves only a few meters, but drives the birds away from the PZ (left of the drone). When the birds discover the drone at a sufficient distance, they adjust their paths horizontally and make smaller changes in the vertical direction. If the drone were able to fly along the flock, it would be possible to herd the birds away from the PZ without breaking the flock.

In Fig. 12, the drone approaches the flock from a shorter distance at a higher speed. In this case, the birds continue to fly to their destination without much horizontal deviation but with large vertical deviation. As the birds discover the approaching drone, they make sudden vertical adjustments to their paths: they rapidly descend by making a very large bank angle with strong flapping motions of their wings. Therefore, if the drone approaches the flock suddenly, the objective of herding may not be satisfied and the flock still continues to fly to the PZ.

From the observations made from Figs. 11 and 12, we can identify the important distance variables shown in Fig. 2. From Fig. $11, R_{\text {fear }}$ is more than $20 \mathrm{~m}$, which corresponds to the distance at which the egrets make horizontal corrections of their paths. From Fig. 12, $R_{\text {agg }}$ is around $10 \mathrm{~m}$ or less. When the birds discover the drone, they choose to make vertical path corrections since they do not have sufficient time to make horizontal corrections by banking. It is also evident from the early 

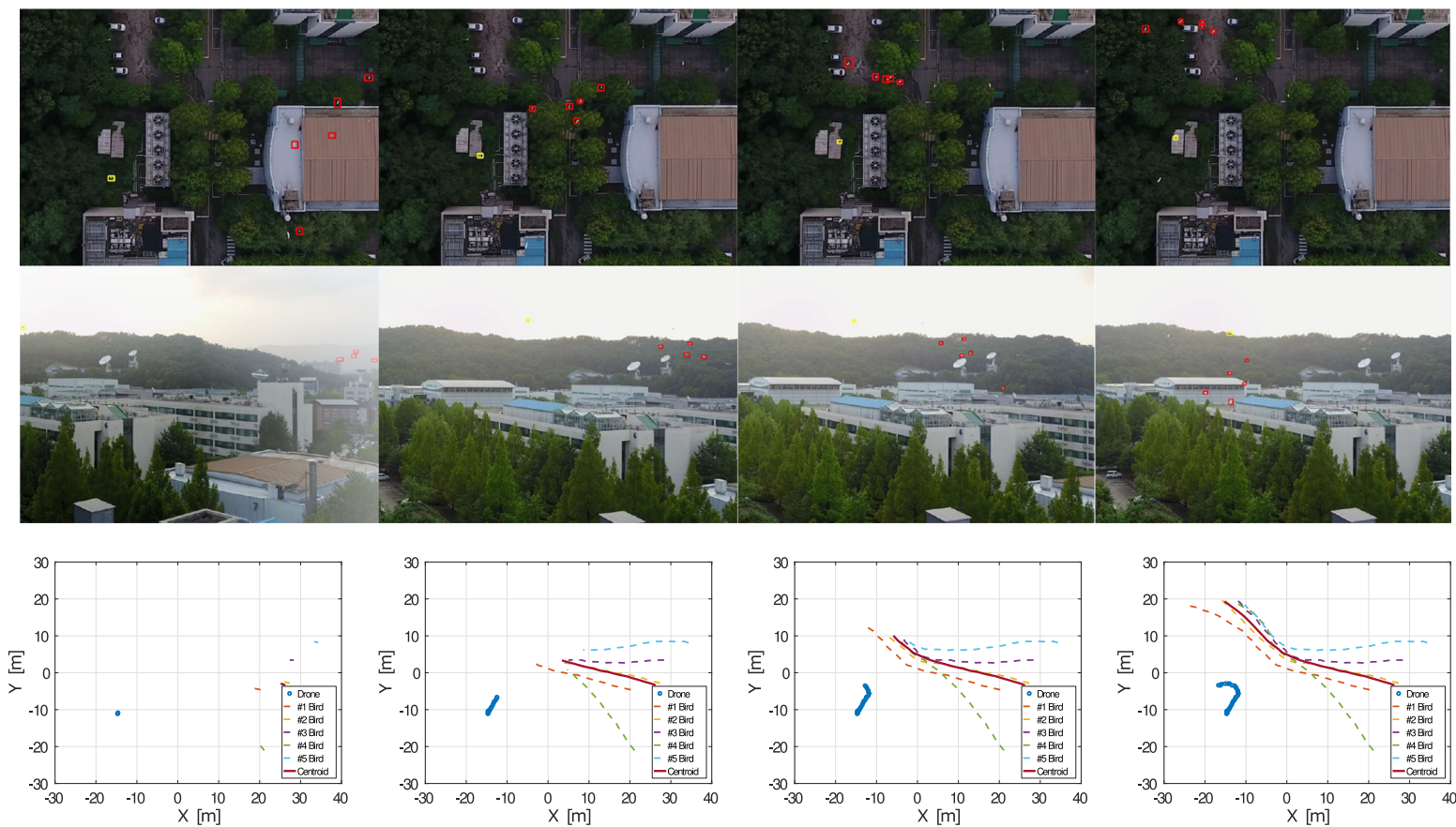

Fig. 11. Stills and data extracted from a video recording of an experiment on live birds. In this experiment, the drone approaches the flock of egrets at a sufficient distance to induce horizontal deviation. The video recording is available as a part of the supplementary material.
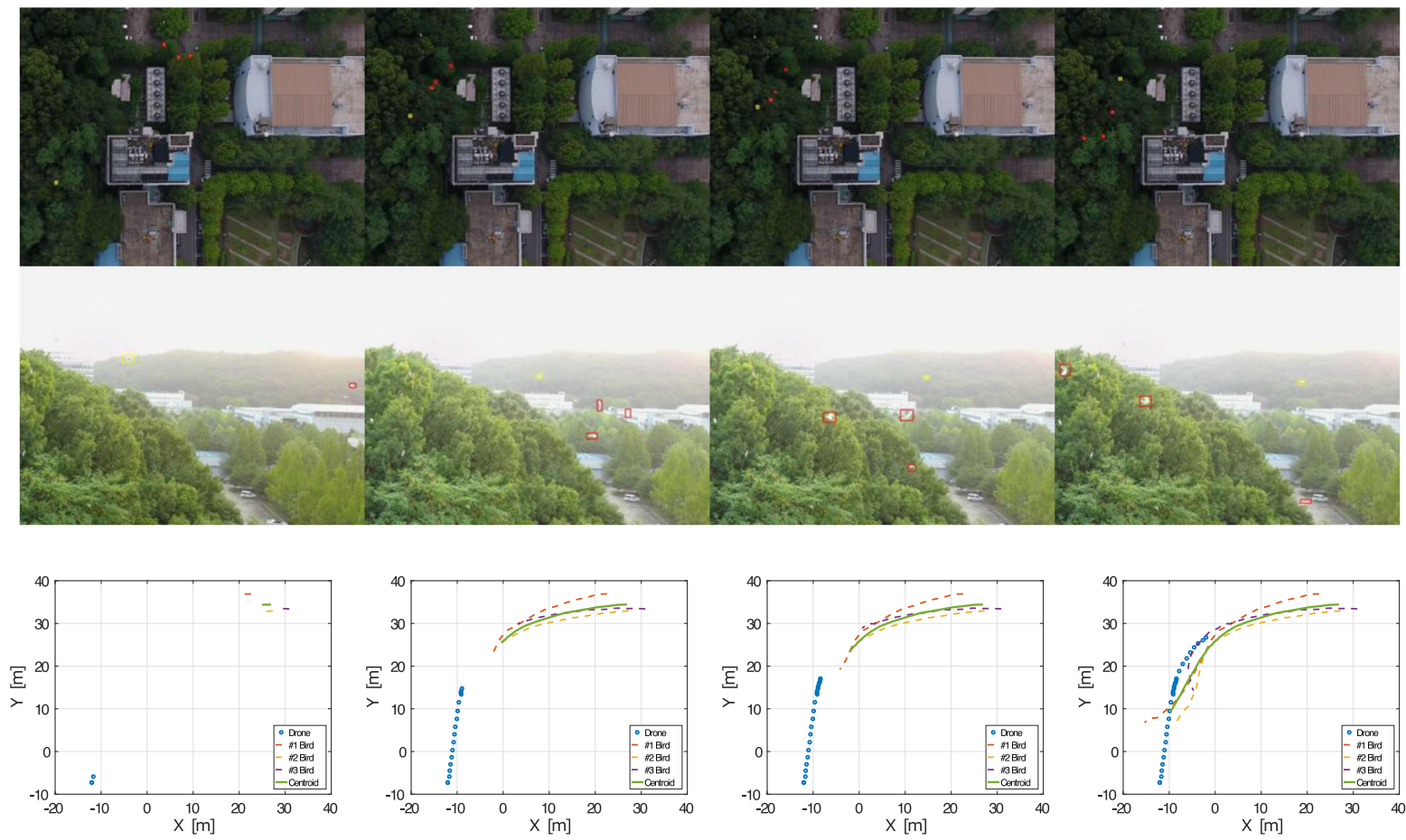

Fig. 12. Stills and data from a video recording of an experiment. The drone approaches the flock of egrets close enough to induce vertical deviation.

moments of Fig. 11 that $R_{\text {safe }}$ is approximately equal to $10 \mathrm{~m}$. The distance between the birds reduces during the engagement with the pursuer, and this behavior is also observed during the simulations described in Section VIII.

The time histories of the position and the velocity of the CG of the flock, corresponding to Fig. 11, are shown in Fig. 13. The time histories of the velocity are estimated from the position data using finite differencing, which explains their coarseness. The velocity profile of the $\mathrm{CG}$ is as follows:

1) until approximately $2.5 \mathrm{~s}$ (pre-engagement): $\left\|\boldsymbol{v}_{c g}(1)\right\|=$ $|\dot{x}|=9 \mathrm{~m} / \mathrm{s} ;\left\|\boldsymbol{v}_{c g}(2)\right\|=\dot{y}=2.5 \mathrm{~m} / \mathrm{s} ;$

2) after $2.5 \mathrm{~s}$ (engagement): $|\dot{x}|=7.5 \mathrm{~m} / \mathrm{s} ; \dot{y}=7.5 \mathrm{~m} / \mathrm{s}$.

It follows from this data that $\boldsymbol{v}_{d}=[-9,2.5,0]$. We recall the dynamics of the velocity of the CG during the engagement 


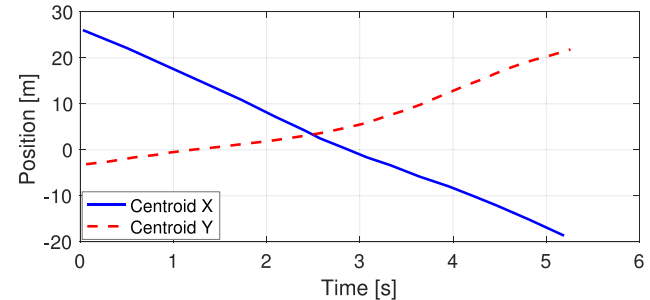

(a)

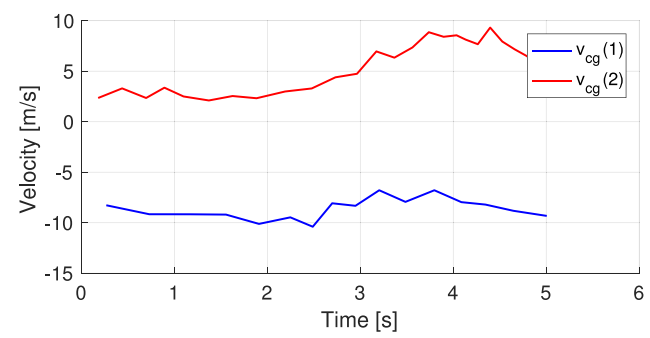

(b)

Fig. 13. Time histories of the position and velocity of the CG of the flock, corresponding to Fig. 11. (a) Position. (b) Velocity.

phase from (19): $\dot{\boldsymbol{v}}_{c g}+k_{g} \boldsymbol{v}_{c g}=k_{g} \boldsymbol{v}_{d}^{\|}$, where $\boldsymbol{v}_{d}^{\|}=\boldsymbol{v}_{d}+$ $\mathbf{F}_{p} / k_{g}$. From the above observation for the velocity of the CG, $\boldsymbol{v}_{d}^{\|}=[-7.5,7.5,0]$. Therefore, we get

$$
\frac{\mathbf{F}_{p}}{k_{g}}=[1.5,5,0] \text {. }
$$

From the time history for the velocity component $\boldsymbol{v}_{c g}(2)(=\dot{y})$, we observe that the time to increase the amplitude by $50 \%$ (from $2.5 \mathrm{~m} / \mathrm{s}$ to $5 \mathrm{~m} / \mathrm{s}$ ) is approximately $1 \mathrm{~s}$. We note that the dynamics of $\boldsymbol{v}_{c g}$ are first-order, and recall a well-known property of the step response of first-order systems that the time to reach 50\% of the amplitude is $\ln (2)$ times the time constant of the system. This allows us to estimate the value of $k_{g}$ as 1.5. Substituting into (21), it follows that $\left\|\mathbf{F}_{p}\right\|=7.8$.

In order to estimate the gain $k_{p}$, we assume that all five birds are engaged by the pursuer. From the plots, the approximate distance between the pursuer and the flock is $15 \mathrm{~m}$. Using this data and (14), we get $\frac{k_{p}}{15^{2}}=7.8 \Rightarrow k_{p}=1755$. Since $k_{g}=1.5$ (from the previous paragraph), we get $k_{p} / k_{g}=1170$.

\section{B. Experiments on Loons}

In the second round of experiments, the drone was required to fly almost parallel to the flight path of a flock of birds, starting from an initial position located just off the nominal flight path. The birds available for this round of experiments had a nominal flight speed that was considerably higher than that of the drone, as a result of which the interaction took place over a relatively short period of time.

Fig. 14 shows the flight paths of several birds and that of the drone. The flight paths shown here were obtained manually from an overhead video (cf., the multimedia supplement), and the coordinates plotted here are measured starting from the top left corner of the video. Upon closer inspection, it was inferred that the birds seen in the video actually comprise several individual flocks (in the sense of connectivity) rather than a single flock. Figure 15 shows three flocks which are clearly discernible, based on the plotted data, together with a phase plot of their mean velocities. The plots in Fig. 15 show only those time instants where the drone was clearly able to interact with the birds.

The data clearly shows the flocks being deflected away from their original flight path as they approach the drone. However, the flight speed of flock 3 changes quite drastically and this variation cannot be captured using the model developed in the paper. Therefore, we confine our analysis to flocks 1 and 2 .

The data obtained from the video does not show the birds returning to their original heading after they cease interacting with the drone. As a result, the gain $k_{g}$ cannot be estimated using the procedure adopted in Section VII-A. As an alternative, we start by estimating the time history of the birds' velocities and accelerations from the data by finite differencing. Next, we employ (14), while assuming that the underlying graph has only bidirectional edges to construct a family of linear equations with $k_{p}$ and $k_{g}$ as unknowns, and solve for them using the linear least squares method.

It turns out that $k_{g} \approx 0$ for both flocks, i.e., they do not show any preferred, predetermined direction of flight. For the flocks labeled 1 and 2 , we get $k_{p} \approx 7000$ and $k_{p} \approx 48000$, respectively. For these two flocks, even if we assume that the underlying graph is directed, the values of $k_{g}$ and $k_{p}$ do not change significantly (although estimates for $k_{s}$ and $k_{a}$ are obtained in the process). This variation across the flocks suggests that the flocking parameters are perhaps a strong function of the flock itself rather than its constituent species or its topology. It is also likely that the evasive response modeled in the paper might be overly simplistic: For instance, the effect of the relative velocity of the drone with respect to the flock or its leader, and the effect of the position of the drone in relation to the flock's velocity vector have been ignored here. It must be noted that flock 2 , which demonstrates a larger $k_{p}$, has the drone more or less directly along its flight path in the horizontal plane.

\section{Summary of the Experimental Results}

Combining the observations in this section with the herding algorithm proposed in Section V, we conclude that the drone should be initially stationed somewhere between the PZ and the approaching flock. If the birds continue to fly toward the PZ, the drone should fly to the flock until it reaches $R_{\text {fear }}$ with a suitable margin. As the birds start to deviate horizontally from the PZ, the drone should follow the flock while maintaining a distance between $R_{\text {fear }}$ and $R_{\text {agg. }}$. If the drone approaches the flock within $R_{\text {agg }}$, the birds may start making vertical path corrections, thereby, causing the birds to fly along their original paths with only vertical deviation. The drone should fly toward the flock at a sufficient distance so that the birds can make horizontal deviation from the original course well in advance. If the drone approaches the flock too abruptly violating $R_{\text {agg }}$, the flock may break or make a sudden vertical adjustment, but still fly towards its original destination and intrude into the PZ. 

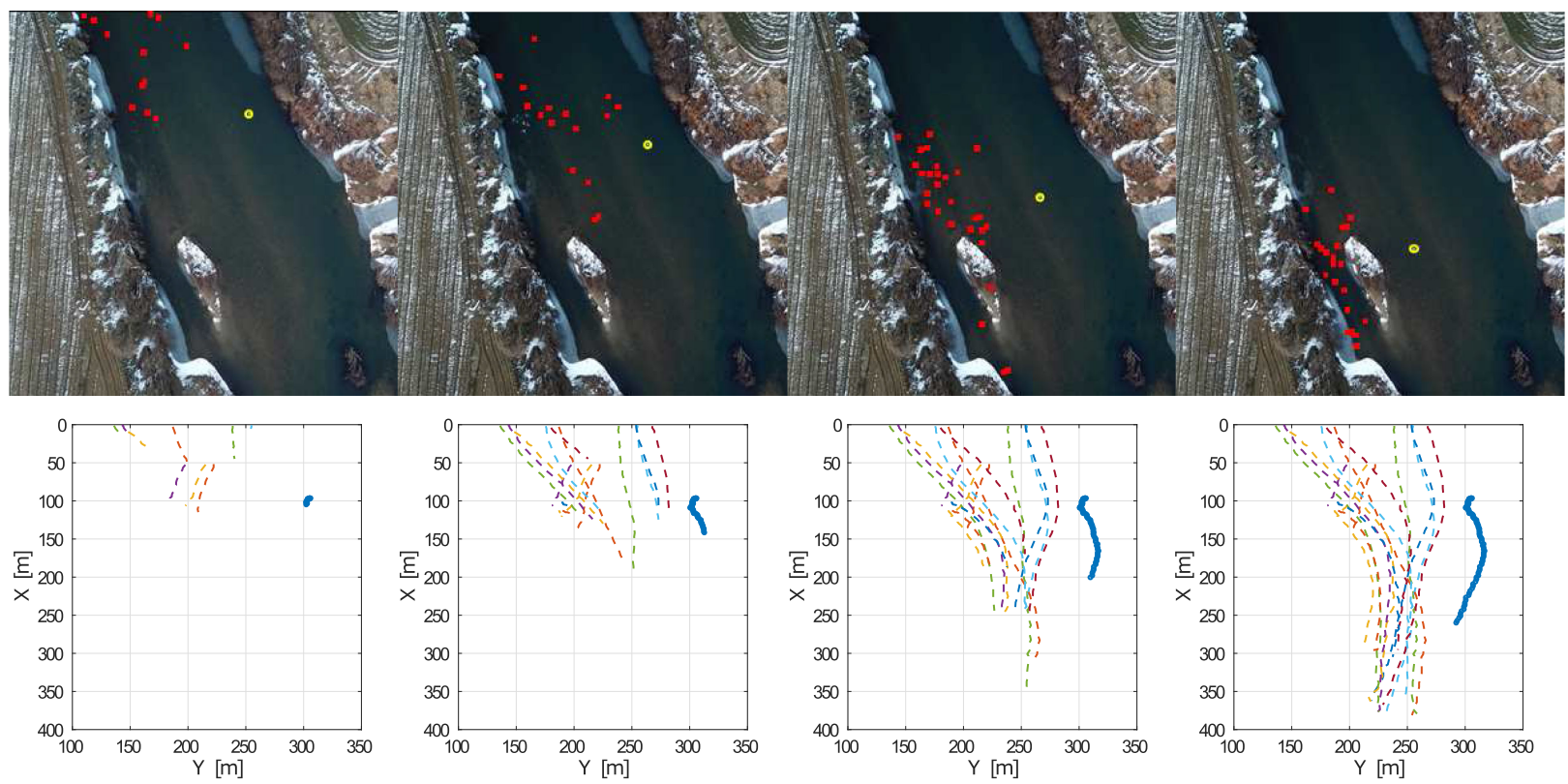

Fig. 14. Stills and data extracted from a video recording of an experiment on live birds. The yellow and red circles denote the drone and the loons, respectively.

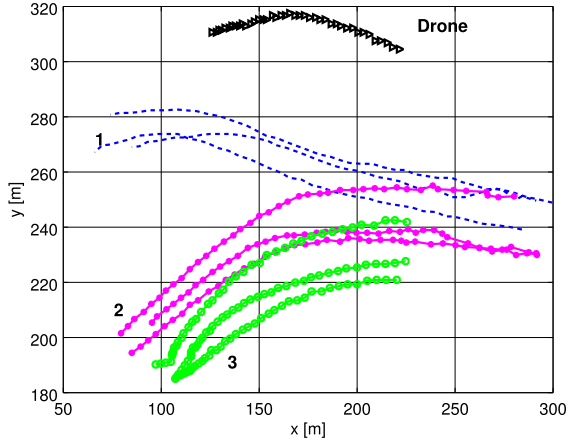

(a)

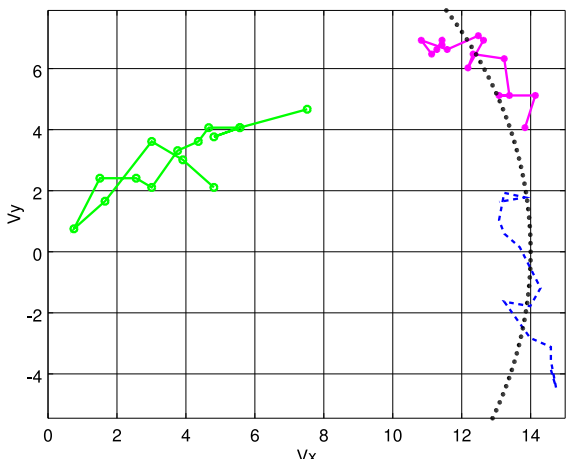

(b)

Fig. 15. Three discernible individual flocks from within the dataset of Fig. 14, and a phase plot of their mean flight velocities. The dotted black curve in the phase plot is a circular arc of radius $14 \mathrm{~m} / \mathrm{s}$. (a) Individual flocks. (b) Velocity phase plot.

\section{SimULATIONS}

Numerical simulations are performed to demonstrate the herding algorithm described in Section V. We consider two cases here: a flock with a regular, dense structure and one with a
TABLE I

FLOCKING MODEL USED FOR SIMULATIONS

\begin{tabular}{|l|l|}
\hline Parameter & Value \\
\hline \hline$R_{\text {safe }}, R_{\text {com }}$ & $5 \mathrm{~m}, 15 \mathrm{~m}$, respectively \\
$R_{\text {fear }}, R_{\text {agg }}$ & $10 \mathrm{~m}, 5 \mathrm{~m}$, respectively \\
$k_{s}, k_{a}, k_{g}, k_{p}$ & $0.02,0.4,0.2,10$, respectively \\
\hline
\end{tabular}

sparse, linear structure. The numbers used to model the flock are listed in Table I. Recall that we derived alternate lower bounds for the minimum approach distance between the pursuer and the flock in Section IV. For the values listed in Table I, the minimum approach distances in (11) and (12) turn out to be $5.34 \mathrm{~m}$ and $6.4 \mathrm{~m}$, respectively, both of which are (slightly) larger than $R_{\mathrm{agg}}$ of $5 \mathrm{~m}$. We initially use a conservative value of $8 \mathrm{~m}$ as the lower bound for the approach distance, and $m=5$ waypoints for the herding algorithm.

Fig. 16 shows the herding of a flock of 16 birds. It is clear that all of the herding objectives are met: the flock is diverted successfully from the airport and the flock radius is bounded at all times. In fact, the flock shrinks slightly during the herding process, as observed in the experiments as well. A minor point of interest is that, while the flock is successfully steered away from $\mathrm{PZ}$, the pursuer itself may end up intruding into $\mathrm{PZ}$ with the current algorithm. A straight-forward solution to this problem is to adjust the distance at which the herding is commenced, since the pursuer needs to be loosely between the flock and the PZ throughout the duration of the herding.

The use of $8 \mathrm{~m}$ as the minimum approach distance turns out to be quite conservative as expected, and we conduct simulations with the minimum distance reduced to $R_{\mathrm{agg}}=5 \mathrm{~m}$. Figures 17 and 18 show the herding of flocks of 16 and 5 birds, respectively, with the reduced approach distance. Notice that the herding distance is reduced by a factor of nearly 2.5 to $270 \mathrm{~m}$ from the 


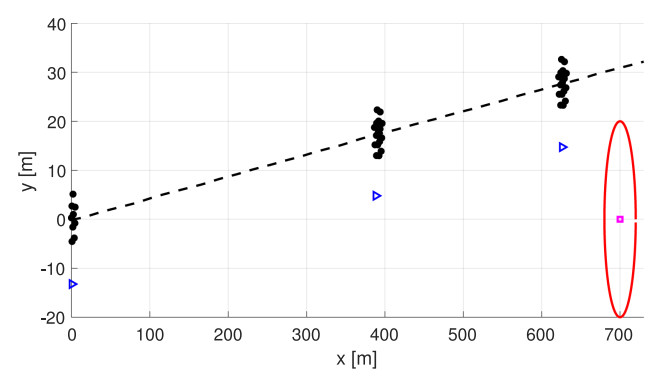

(a)

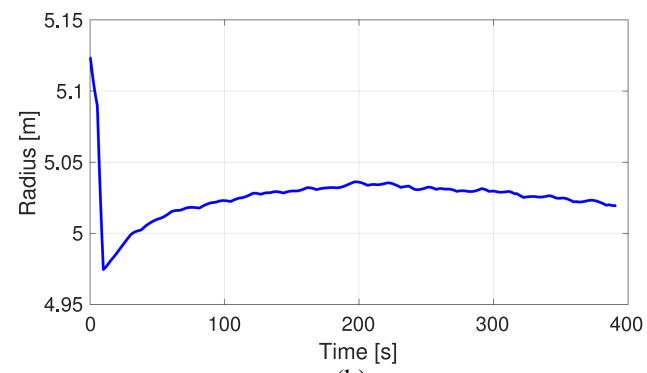

(b)

Fig. 16. Simulation showing the herding of a flock with 16 birds and minimum approach distance of $8 \mathrm{~m}$. (a) Snapshots and CG track (dashed curve). (b) Time history of flock radius.

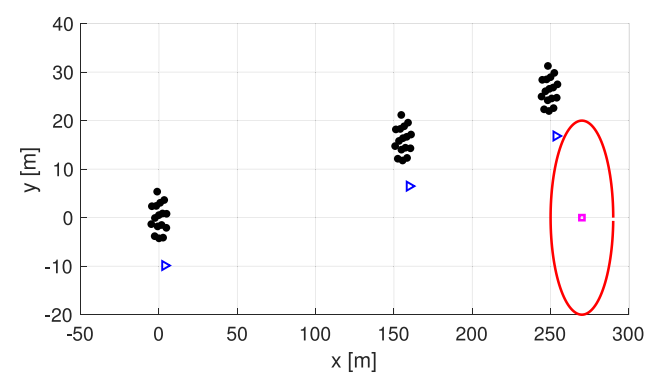

Fig. 17. Simulation showing the herding of a flock with 16 birds with reduced approach distance.

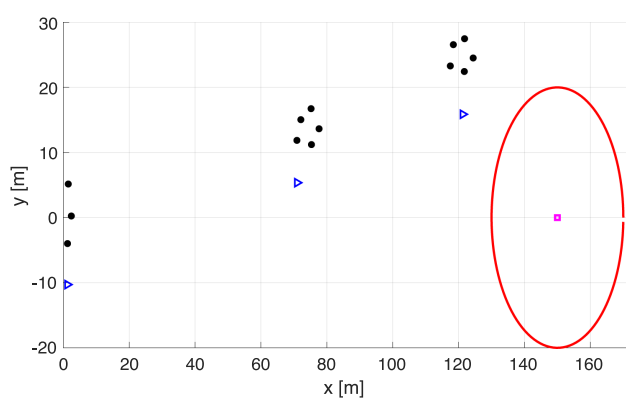

Fig. 18. Simulation showing the herding of a flock with 5 birds with reduced approach distance.

initial value of $700 \mathrm{~m}$ seen in Fig. 16. Furthermore, the flock radius changes very little during the herding of 16 birds. For the case of 5 birds, we start the simulation with a flock which is almost linear at the outset. Notice that the flock converges to an approximate pentagon as the herding proceeds, which is accompanied by a marked reduction in the flock radius.
Using the plots shown here, we can estimate, for the modeling parameters in Table I, the minimum distance required to achieve a $30 \mathrm{~m}$ lateral shift in flocks of sizes different than those considered here. Since the distance is almost $270 \mathrm{~m}$ for a flock of 16 birds, we use the approximation of Remark 1 to estimate that $D_{p, \min }(n) \approx 17 n$ for large $n$. On the other hand, since the pursuer needs $150 \mathrm{~m}$ for a flock of 5 birds, it is reasonable to expect $150 \mathrm{~m}$ as the minimum herding distance for a small flock. These calculations also suggest that a flock size of 9 forms a threshold between small and large flocks for the purpose of estimating the herding distances.

\section{CONCLUDING Discussion}

We investigated the problem of diverting a flock of birds away from a prescribed area, such as an airport, using a robotic UAV. A novel boundary control strategy, called the $m$-waypoint algorithm, was introduced in the paper for enabling a single pursuer UAV to safely herd the flock without fragmenting it. We derived conditions under which flocks are exponentially stable to external perturbations. Using geometric techniques, approximate analytical expressions were derived for the safe approach distance between a pursuer and a flock. The performance of the herding algorithm was examined systematically to obtain an expression for the minimum distance from the airport at which the herding needs to be commenced. An important contribution of the paper is the reporting of experiments, conducted on live birds, to estimate the parameters of the flocking model and verify the potential of the herding algorithm. Numerical simulation results were performed to demonstrate the herding algorithm on representative flocks.

The flocking model, described by (4) and (5), is based on wellknown models in the literature. To the best of our knowledge, the work reported in Section VII is the first instance of parameter identification in a flocking model using experimental data. The identification of the parameters $k_{s}$ and $k_{a}$ in (4), and the factors that determine them, is an open problem.

The herding algorithm in this paper was developed on the basis of certain macroscopic properties of the flocking model (14) and a known model for the evasive response of the flock. Results from Section VII-B suggest that (5) needs to be refined before it can be deemed as an accurate representation for a flock's response. More specifically, we need to determine the factors that determine the gain $k_{p}$, and further experiments on live birds are needed for that purpose and for a conclusive validation of the herding algorithm.

\section{ACKNOWLEDGMENT}

The authors would like to thank Shripad Gade for his contributions to the initial phase of this project, and the anonymous reviewers for their insightful comments.

\section{REFERENCES}

[1] J. R. Allan, "The costs of bird strike and bird strike prevention," in $\mathrm{Hu}$ man Conflicts with Wildlife: Economic Considerations, Proceedings of the Third NWRC Special Symposium, L. Clark, Ed. Fort Collins, CO, USA: National Wildlife Research Center, 2000. 
[2] N. S. Sodhi, "Competition in the air: Birds versus aircraft," The Auk, vol. 119 , no. 3, pp. 587-595, 2002.

[3] National Wildlife Strike Database, "Wildlife strikes to civil aircraft in the United States 1990-2013,” Federal Aviation Admin., Washington, DC, USA, Serial Report Number 20, Jul. 2014.

[4] International Bird Strike Committee, "Standards for aerodrome bird/wildlife control," Recommended Practices No. 1, 2006.

[5] R. E. Harris and R. A. Davis, "Evaluation of the efficacy of products and techniques for airport bird control," LGL Limited Aerodrome Safety Branch, King City, ON, Canada, 1998.

[6] Clear Flight Solutions. [Online]. Available: http://clearflightsolutions. com/, accessed: Dec 20, 2014.

[7] S. Gade, A. A. Paranjape, and S.-J. Chung, "Herding a flock of birds approaching an airport using an unmanned aerial vehicle," in Proc. AIAA Guid. Navig. Control Conf., Kissimmee, FL, 2015, AIAA Paper 2015-1540.

[8] S. Gade, A. A. Paranjape, and S.-J. Chung, "Robotic herding using wavefront algorithm: Performance and stability," in Proc. AIAA Guid. Navig. Control Conf., San Diego, CA, 2016, AIAA Paper 2016-1378.

[9] C. W. Reynolds, "Flocks, herds and schools: A distributed behavioral model," ACM SIGGRAPH Comput. Graph., vol. 21, no. 4, pp. 25-34, 1987.

[10] T. Vicsek, A. Czirók, E. Ben-Jacob , I. Cohen, and O. Shochet, "Novel type of phase transition in a system of self-driven particles," Phys. Rev. Lett., vol. 75, pp. 1226-1229, 1995.

[11] A. Czirók and T. Vicsek, "Collective behavior of interacting self-propelled particles," Physica A, Stat. Mech. Appl., vol. 281, no. 1, pp. 17-29, 2000.

[12] C. Hartman and B. Benes, "Autonomous boids," Comput. Animat. Virtual Worlds, vol. 17, no. 3/4, pp. 199-206, 2006.

[13] M. Ballerini et al., "Empirical investigation of starling flocks: A benchmark study in collective animal behaviour," Animal Behav., vol. 76, no. 1, pp. 201-215, 2008.

[14] M. Ballerini et al., "Interaction ruling animal collective behavior depends on topological rather than metric distance: Evidence from a field study," in Proc. Nat. Acad. Sci., vol. 105, no. 4, pp. 1232-1237, 2008.

[15] D. J. Sumpter, Collective Animal Behavior. Princeton, NJ, USA: Princeton Univ. Press, 2010

[16] R. Olfati-Saber and R. M. Murray, "Consensus problems in networks of agents with switching topology and time-delays," IEEE Trans. Autom. Control, vol. 49, no. 9, pp. 1520-1533, Sep. 2004.

[17] R. Olfati-Saber, "Flocking for multi-agent dynamic systems: Algorithms and theory," IEEE Trans. Autom. Control, vol. 51, no. 3, pp. 401-420, Mar. 2006.

[18] H. G. Tanner, A. Jadbabaie, and G. J. Pappas, "Flocking in fixed and switching networks," IEEE Trans. Autom. Control, vol. 52, no. 5, pp. $863-$ 868, May 2007.

[19] A. Jadbabaie, J. Lin, and A. S. Morse, "Coordination of groups of mobile autonomous agents using nearest neighbor rules," IEEE Trans. Autom. Control, vol. 48, no. 6, pp. 988-1001, Jun. 2003.

[20] Q.-C. Pham and J.-J. E. Slotine, "Stable concurrent synchronization in dynamic system networks," Neural Netw., vol. 20, no. 1, pp. 62-77, 2007.

[21] S.-J. Chung and J.-J.E. Slotine, "Cooperative robot control and concurrent synchronization of lagrangian systems," IEEE Trans. Robot., vol. 25, no. 3, pp. 686-700, Jun. 2009.

[22] S.-J. Chung, S. Bandyopadhyay, I. Chang, and F. Y. Hadaegh, "Phase synchronization control of complex networks of lagrangian systems on adaptive digraphs," Automatica, vol. 49, no. 5, pp. 1148-1161, 2013.

[23] J. Toner and Y. Tu, "Long-range order in a two-dimensional dynamical XY model: How birds fly together," Phys. Rev. Lett., vol. 75, no. 3, pp. 43264329, 1995

[24] D. V. Dimarogonas and K. H. Johansson, "Further results on the stability of distance-based multi-robot formations," in Proc. Amer. Control Conf., 2009, pp. 2972-2977.

[25] D. V. Dimarogonas and K. H. Johansson, "On the stability of distancebased formation control," in Proc. 47th IEEE Conf. Decis. Control, 2008, pp. $1200-1205$.

[26] L. Moreau, "Stability of continuous-time distributed consensus algorithms," in Proc. 43rd IEEE Conf. Decis. Control, 2004, pp. 39984003.

[27] J. A. Carrillo, Y. Huang, and S. Martin, "Nonlinear stability of flock solutions in second-order swarming models," Nonlinear Anal.: Real World Appl., vol. 17, pp. 332-343, 2014.
[28] M. Zheng, Y. Kashimori, O. Hoshino, K. Fujita, and T. Kambara, "Behavior pattern (innate action) of individuals in fish schools generating efficient collective evasion from predation," J. Theoretical Biol., vol. 235, no. 2, pp. 153-167, 2005.

[29] S.-H. Lee, H. Pak, and T.-S. Chon, "Dynamics of prey-flock escaping behavior in response to predator's attack," J. Theoretical Biol., vol. 240, no. 2, pp. 250-259, 2006.

[30] A. Procaccini et al., "Propagating waves in starling, sturnus vulgaris, flocks under predation," Animal Behav., vol. 82, no. 4, pp. 759-765, 2011.

[31] J. E. Herbert-Read, J. Buhl, F. Hu, A. J. Ward, and D. J. Sumpter, "Initiation and spread of escape waves within animal groups," Roy. Soc. Open Sci., vol. 2, no. 4, 2015, Art no. 140355.

[32] C. K. Hemelrijk, L. van Zuidam, and H. Hildenbrandt, "What underlies waves of agitation in starling flocks," Behav. Ecology Sociobiol., vol. 69, no. 5, pp. 755-764, 2015.

[33] N. O. Handegard, K. M. Boswell, C. C. Ioannou, S. P. Leblanc, D. B. Tjøstheim, and I. D. Couzin, "The dynamics of coordinated group hunting and collective information transfer among schooling prey," Current Biol., vol. 22, no. 13, pp. 1213-1217, 2012.

[34] C. Carere et al., "Aerial flocking patterns of wintering starlings, sturnus vulgaris, under different predation risk," Animal Behav., vol. 77, no. 1, pp. 101-107, 2009.

[35] R. Vaughan, N. Sumpter, J. Henderson, A. Frost, and S. Cameron, "Experiments in automatic flock control," Robot. Auton. Syst., vol. 31, no. 1, pp. 109-117, 2000.

[36] J.-M. Lien, B. Bayazit, R. T. Sowell, S. Rodriguez, and N. M. Amato, "Shepherding behaviors," in Proc. IEEE Int. Conf. Robot. Automat., 2004 vol. 4, pp. 4159-4164.

[37] J.-M. Lien, S. Rodriguez, J.-P. Malric, and N. M. Amato, "Shepherding behaviors with multiple shepherds," in Proc. IEEE Int. Conf. Robot. Autom. 2005, pp. 3402-3407.

[38] M. Haque, A. Rahmani, and M. Egerstedt, "A hybrid, multi-agent model for foraging bottlenose dolphins," in Proc. Anal. Des. Hybrid Syst., University Zaragoza, Spain, 2009, pp. 262-267.

[39] M. A. Haque, A. R. Rahmani, and M. B. Egerstedt, "Biologically inspired confinement of multi-robot systems," Int. J. Bio-Inspired Comput., vol. 3, no. 4 , pp. 213-224, 2011

[40] D. Strömbom et al., "Solving the shepherding problem: Heuristics for herding autonomous, interacting agents," J. Roy. Soc. Interface, vol. 11, no. 100, 2014, Art. no. 20140719.

[41] T. J. Nohara, P. Weber, A. Ukrainec, A. Premji, and G. Jones, "An overview of avian radar developments-Past, present and future," Bird Strike Committee Proc., Kingston, ON, 2007, Paper 13.

[42] J. Carr, Applications of Center Manifold Theory. New York, NY, USA: Springer, 1981

[43] A. A. Paranjape, K. C. Meier, X. Shi, S.-J. Chung, and S. Hutchinson, "Motion primitives and 3D path planning for fast flight through a forest," Int. J. Robot. Res., vol. 34, no. 3, pp. 357-377, Mar. 2015.

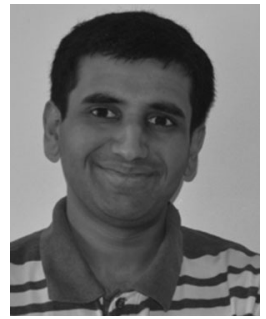

Aditya A. Paranjape received the B.Tech and M.Tech degrees in aerospace engineering from the Indian Institute of Technology Bombay, Mumbai, India, in 2007, and the Ph.D. degree in aerospace engineering from the University of Illinois at UrbanaChampaign, Urbana, IL, USA, in 2011.

$\mathrm{He}$ is currently a Lecturer in aerial robotics with Imperial College London, London, U.K., and an Adjunct Professor with the Department of Mechanical Engineering, McGill University, Montreal, QC, Canada. His research interests include dynamics and control of aerospace vehicles, infinite dimensional systems, and multiagent systems. 


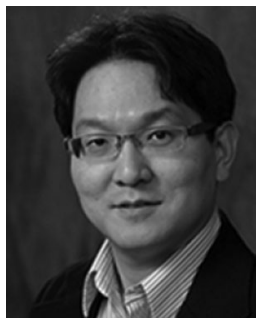

Soon-Jo Chung (M'06-SM'12) received the B.S. degree in aerospace engineering (summa cum laude) from the Korea Advanced Institute of Science and Technology, Daejeon, South Korea, in 1998, and the S.M. degree in aeronautics and astronautics, and the Sc.D. degree in estimation and control from the Massachusetts Institute of Technology, Cambridge, MA, USA, in 2002 and 2007, respectively.

$\mathrm{He}$ is an Associate Professor of aerospace and a Bren Scholar and Jet Propulsion Laboratory Research Scientist with the California Institute of Technology, Pasadena, CA, USA. From 2009 to 2016, he was on the faculty of the University of Illinois at Urbana-Champaign (UIUC), Urbana, IL, USA. His research interests include spacecraft and aerial swarms and autonomous aerospace systems, and in particular, the theory and application of complex nonlinear dynamics, control, estimation, guidance, and navigation of autonomous space and air vehicles.

Dr. Chung is the recipient of the UIUC Engineering Deans Award for Excellence in Research, the Beckman Faculty Fellowship of the UIUC Center for Advanced Study, the U.S. Air Force Office of Scientific Research Young Investigator Award, the National Science Foundation Faculty Early Career Development Award, and three Best Conference Paper Awards from the IEEE, and the American Institute of Aeronautics and Astronautics. He is an Associate Editor for the IEEE TRANSACTIONS ON ROBOTICS and the Journal of Guidance, Control, and Dynamics (AIAA).

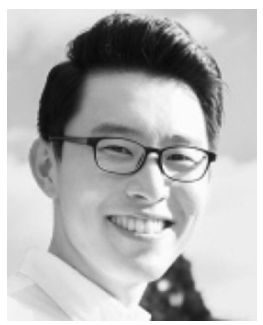

Kyunam Kim received the B.S. degree (summa cum laude) in mechanical and aerospace engineering from Seoul National University, Seoul, South Korea, in 2010, and the M.S. and Ph.D. degrees in mechanical engineering from the University of California at Berkeley, Berkeley, CA, USA, in 2012 and 2016, respectively.

He is currently a Postdoctoral Scholar with the Department of Aerospace, California Institute of Technology, Pasadena, CA, USA. His research interests include nonlinear dynamics, control, and estimation, and their application to novel robotic systems in the aerospace field.

Dr. Kim was the recipient of Samsung Scholarship from 2010 to 2015.

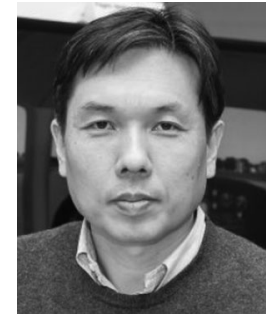

David Shim received the B.S. and M.S. degrees in mechanical design and production engineering from Seoul National University, Seoul, South Korea, in 1991 and 1993, respectively, and the Ph.D. degree in mechanical engineering from the University of California at Berkeley, Berkeley, CA, USA, in 2000.

He was with Hyundai Motor Company and Maxtor Corporation. In 2007, he joined the Department of Aerospace Engineering, KAIST, Daejeon, South Korea, and is currently a tenured Associate Professor with the Department of Electrical Engineering, KAIST. His research interests include control system, autonomous vehicles, and robotics. He is also a Director of Korea Civil RPAS Research Center. 